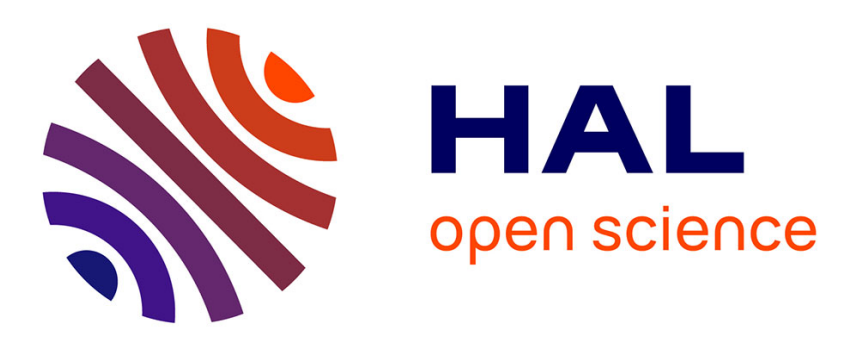

\title{
Numerical simulation of the viscous shock tube problem by using a high resolution monotonicity-preserving scheme
}

\author{
Virginie Daru, Christian Tenaud
}

\section{- To cite this version:}

Virginie Daru, Christian Tenaud. Numerical simulation of the viscous shock tube problem by using a high resolution monotonicity-preserving scheme. Computers and Fluids, 2009, 38 (3), pp.664-676. 10.1016/j.compfluid.2008.06.008 . hal-01400427

\section{HAL Id: hal-01400427 \\ https://hal.science/hal-01400427}

Submitted on 5 Sep 2017

HAL is a multi-disciplinary open access archive for the deposit and dissemination of scientific research documents, whether they are published or not. The documents may come from teaching and research institutions in France or abroad, or from public or private research centers.
L'archive ouverte pluridisciplinaire HAL, est destinée au dépôt et à la diffusion de documents scientifiques de niveau recherche, publiés ou non, émanant des établissements d'enseignement et de recherche français ou étrangers, des laboratoires publics ou privés. 


\title{
Numerical simulation of the viscous shock tube problem by using a high resolution monotonicity-preserving scheme.
}

\author{
Virginie Daru ${ }^{(a, b)}$ and Christian Tenaud ${ }^{(a)}$ \\ ${ }^{a}$ LIMSI-UPR CNRS 3251, BP 133, 91403 ORSAY Cedex, FRANCE \\ Virginie.Daru@limsi.fr and Christian.Tenaud@limsi.fr \\ ${ }^{\mathrm{b}}$ ENSAM, Lab. SINUMEF, 151 Bd de l'Hopital, 75013 PARIS, FRANCE
}

\begin{abstract}
This work deals with the flow generated in a shock tube after the shock wave has reflected at the end wall. For a viscous fluid, a complex unsteady interaction takes place between the incident boundary layer and reflected shock wave. The numerical simulation of this complex flow requires both robust and accurate numerical schemes. In this work, we rely on the one step high order scheme recently proposed in [4]. With this scheme, converged results are obtained for Reynolds numbers in the range 200 to 1000 . The interaction mechanisms are carefully analyzed as well as the flow dynamics.
\end{abstract}

Key words: High-resolution shock capturing scheme, compressible flow, shock tube problem, shock-wave boundary-layer interaction

\section{Introduction}

The interaction of a moving shock wave with a boundary layer is encountered in many flow configurations (buffet, air intakes, over-expanded nozzles, shock tubes). These configurations exhibit unsteady boundary layer detachment over large spatial extents which are responsible for unsteady aerodynamic loads and fluid mixing spoiling the system and dramatically reducing the performance. A fine understanding of the physical mechanisms responsible for unsteadiness, requires the development of very accurate numerical methods for the simulation of these flows, with a large number of grid-points. Therefore, low cost numerical schemes are called to obtain grid-converged solutions at an acceptable CPU time. 
A model configuration that exhibits these phenomena corresponds to the shock tube problem. It can be found in various real apparatus, such as in high speed aerodynamic flow facilities used to study the chemical relaxation process in high temperature gas mixtures or to generate high enthalpy reservoir conditions. In flow facility configurations, the free piston reflected shock tunnel is ended by a nozzle communicating with the test section. However, the premature contamination of the flow in the test section, through the production of intense vortices by interaction of the reflected shock-wave and the end wall, limits the usefulness of such devices.

In fact, the reflected shock wave interacts with the incident boundary layer with modified gas properties in this end wall region as a result: a complex unsteady interaction develops between the reflected shock wave and the boundary layer. When the stagnation pressure within the boundary layer is lower than that downstream of the normal reflected shock wave, the boundary layer separates and a lambda-like shock wave pattern forms. This flow configuration has been first analyzed by Mark [13] using a crude model. Experiments were also carried out allowing the verification of the theoretical model in [13]. Other detailed experimental analyses of the shock-wave / boundary layer interaction in a shock-tube were presented in [7, 14], leading to enhancements of Mark's model.

The numerical simulation of shock-wave boundary layer interaction in a shocktube was also studied numerically by several authors over the last decade. The first computations were performed by Weber et al. [21] who analyzed the interaction of an isolated reflected shock-wave with a laminar boundary layer; in this work, the contact discontinuity and rarefaction waves present in a shock-tube were disregarded. Other numerical studies focused on the flow contamination at the end-wall of the shock-tube $[1,8]$. The shock-tube configuration at moderate Reynolds number also served as a test-case for numerical method validation $[3,6,10,11,19]$. The numerical simulation of this type of flow requires numerical schemes which are both robust and highly accurate, to simultaneously represent with high precision the smooth regions of the flow and capture the discontinuities. In a previous study [3], we exhibited the convergence issues encountered when using classical shock capturing TVD approaches on such a test-case. Similar conclusions were also drawn by Sjögreen and Yee [19] for ACM, TVD or MUSCL approaches. We recently developed an original high order coupled time-space scheme (named OSMP7, accurate up to the 7th-order, in the scalar case) which guaranties Monotonicity Preserving (MP) properties [4, 5]. The OSMP7 scheme gives very accurate results on numerous classical test cases of 2-D free supersonic flows [4], [5] and on aeroacoustic problems [2]. Compared to more classical schemes (RungeKutta time integration and ENO or WENO space discretization), the OSMP7 scheme better captures the discontinuities, for comparable order of accuracy in the smooth regions. However, the OSMP7 scheme is six time less expensive 
than the RK3-WENO schemes in terms of CPU times.

The objective of this paper is two fold. First, we apply the higher-order accurate OSMP7 scheme to the shock tube problem for several Reynolds numbers ( $R e=200,500,750$ and 1000). We have restricted ourselves to this range of Reynolds numbers to limit the grid size requirements. The OSMP7 scheme allows for highly converged results, essentially grid independent, that could not be obtained previously with other schemes [3]. The results are confronted with those of Sjögreen and Yee [19] for comparable computational meshes. Secondly, we provide a detailed analysis of the shock-wave / boundary-layer interaction and flow dynamics inside the shock tube.

The paper is organized as follows. After a brief review of the governing equations, we present in section (2) the different numerical approaches used in this work: the method of lines (based on Runge-Kutta time integration coupled with a WENO space discretization) and the so-called OSMP (based on coupled time and space integration with MP constraint). The flow field configuration of the shock tube problem is presented in section (3). Section (4) is devoted to a study of the influence of the numerical parameters, grid convergence assessment and the influence of the different numerical discretization schemes. The dynamics of the shock wave / boundary layer interaction is analyzed in section (5). The Reynolds number is the key parameter of the study and its influence on the dynamics is detailed in section (6). Conclusions and comments are summarized in section (7).

\section{Numerical procedure}

\subsection{The set of equations}

The governing equations are the compressible Navier-Stokes equations. In conservative form and Cartesian coordinates, they write as:

$$
\mathbf{w}_{t}+\nabla \cdot\left(\mathbf{f}(\mathbf{w})-\mathbf{f}^{v}(\mathbf{w}, \nabla \mathbf{w})\right)=0
$$

where $\mathbf{w}=(\rho, \rho \mathbf{u}, \rho E)^{t}$ is the vector of the conservative variables, using the classical notations, and $\mathbf{f}(\mathbf{w})$ and $\mathbf{f}^{v}$ are the Euler and the viscous fluxes re- 
spectively:

$$
\begin{gathered}
\mathbf{f}=\left(\begin{array}{c}
\rho \mathbf{u} \\
\rho \mathbf{u} \otimes \mathbf{u}+\frac{P}{\gamma M_{0}^{2}} \mathbb{I} \\
\rho \mathbf{u} E+\mathbf{u} \frac{P}{\gamma M_{0}^{2}}
\end{array}\right), \\
\mathbf{f}^{v}=\left(\begin{array}{c}
0 \\
\sigma \\
\mathbf{u} \cdot \sigma+\mathbf{\Psi}
\end{array}\right),
\end{gathered}
$$

with the strain rate tensor

$$
\sigma=\frac{\mu}{\operatorname{Re}}\left(\nabla \mathbf{u}+\nabla^{t} \mathbf{u}-\frac{2}{3} \nabla \cdot \mathbf{u} \mathbb{I}\right)
$$

and the heat flux

$$
\Psi=\frac{\mu}{(\gamma-1) \operatorname{Re} \operatorname{Pr} \mathrm{M}_{0}^{2}} \nabla T
$$

In addition, a perfect gas law is used:

$$
\begin{aligned}
\frac{P}{\gamma M_{0}^{2}} & =(\gamma-1)\left[\rho E-\frac{1}{2} \rho \mathbf{u} \cdot \mathbf{u}\right], \\
T & =\frac{P}{\rho},
\end{aligned}
$$

with $\rho$ the fluid density, $\mathbf{u}$ the velocity vector, $P$ the static pressure, $T$ the static temperature, $E$ the total energy per unit of mass and $\mu$ the dimensionless dynamic viscosity.

These equations are written in dimensionless form by using the reference values of the density $\left(\rho_{0}\right)$, the velocity $\left(v_{0}\right)$, and the length scale $\left(L_{0}\right)$. The Reynolds number is based on the reference values: $\mathrm{Re}=\rho_{0} \mathrm{v}_{0} \mathrm{~L}_{0} / \mu\left(\mathrm{T}_{0}\right)$. The Mach number is $M_{0}=v_{0} / \sqrt{\gamma \mathrm{RT}_{0}}$ with $\mathrm{R}=287 \mathrm{~J} . \mathrm{Kg}^{-1} \cdot \mathrm{K}^{-1}$ (for air) the gas constant.

For simplicity, this study considers an ideal gas (air) with constant specific heat ratio $\gamma=1.4$, constant dynamic viscosity $\mu=1$ and constant Prandtl number $\operatorname{Pr}=0.73$. 


\subsection{Space and time integrations}

The resolution of the Navier-Stokes equations $(1,2,3)$ rely on a finite volume approach. The viscous fluxes (3) are discretized by means of a second-order accurate centered scheme, whatever coupled or decoupled time and space integrations are. For the coupled time and space approach, details on this viscousflux discretization can be found in Daru and Tenaud [3]. To check the influence of the viscous flux discretization order on the solution, we also performed few simulations using a fourth-order accurate centered scheme. It was found that the viscous scheme has negligible influence on the resulting solutions (not shown). Therefore, all presented results correspond to the centered 2nd-order viscous flux discretization.

Regarding the convective fluxes, two numerical approximations have been compared in this study. The first one is the high-order coupled time and space scheme proposed in $[5,6]$. The second one, using splitted time and space integrations, is a "Method of lines" approach, based on a multi-stage (Runge-Kutta) time integration. In each sub-stage of the time integration, a high order spatial (WENO) dicretization is applied.

This study is conducted on cartesian meshes. We denote by $w_{j}^{n}$ the discrete quantity $w(\mathbf{x}, t)$ estimated at a grid point $\mathbf{x}_{j}=j \delta \mathbf{x}$ and at time $t=n \delta t, \delta t$ and $\delta \mathbf{x}$ being, respectively, the time and space steps.

\subsubsection{Coupled time and space approach}

The unlimited One-Step scheme developed in [5] is of Lax-Wendroff type [12]. These schemes are constructed in the scalar case by correcting the error terms of the successive modified equations, to yield one additional order of accuracy at each level. For stability reasons, the error term, involving highest degree derivatives at each level, is discretized using upwind formulae for odd derivatives and centred formulae for even derivatives. In this way, one obtains a recurrence relation to construct schemes with arbitrary order of accuracy in time and space.

In the non-linear system case, the accuracy order cannot be maintained easily as a Cauchy-Kowalewski procedure is required along the derivation of the successive modified equations. Here, a simpler procedure is used, consisting in a local linearization on the eigenvector basis at the cells' interfaces.

To illustrate the procedure, let us consider a 1-D system of equations $\mathbf{w}_{t}+$ $\nabla \cdot \mathbf{f}(\mathbf{w})=0$, and define $\lambda_{k}$ and $\mathbf{r}_{k}$ the eigenvalues and right eigenvectors of the Roe-averaged jacobian matrix $A=\left(\frac{d f}{d w}\right)_{j+1 / 2}$ centered on the inter- 
face $(j+1 / 2), \delta \alpha_{k, j+1 / 2}=\mathbf{r}_{k} \cdot\left(w_{j+1}^{n}-w_{j}^{n}\right)$ the $k$-th Riemann invariant, and $\nu_{k, j+1 / 2} \frac{\delta t}{\delta x} \lambda_{k, j+1 / 2}$ the local CFL number.

The one-step scheme reads:

$$
w_{j}^{n+1}=w_{j}^{n}-\frac{\delta t}{\delta x}\left(F_{j+1 / 2}^{p}-F_{j-1 / 2}^{p}\right)
$$

where $F_{j+1 / 2}^{p}$ is the $p$ th-order accurate numerical flux of the scheme at the cell interface $(j+1 / 2)$, which can be written:

$$
F_{j+1 / 2}^{p}=F_{j+1 / 2}^{R o e}+\frac{1}{2} \sum_{k}\left(\psi^{p} \mathbf{r}\right)_{k, j+1 / 2}
$$

For clarity, the superscript $n$ has been omitted in the expression of the fluxes. $F_{j+1 / 2}^{R o e}$ is the first order Roe flux defined as follows:

$$
F_{j+1 / 2}^{R o e}=\frac{1}{2}\left(f_{j}+f_{j+1}\right)-\frac{1}{2} \sum_{k}(\delta|f| \mathbf{r})_{k, j+1 / 2},
$$

with

$$
\delta|f|_{k, j+1 / 2}=|\lambda|_{k, j+1 / 2} \delta \alpha_{k, j+1 / 2}
$$

The function $\psi$ can be decomposed in odd, corresponding to the odd derivatives in the modified equations, and even parts:

$$
\psi_{k, j+1 / 2}^{p}=\sum_{n=1}^{m} \psi_{k, j+1 / 2}^{2 n}-j s \sum_{n=1}^{m 1} \psi_{k, j+1-j s / 2}^{2 n+1}
$$

where $m=\lfloor p / 2\rfloor, m 1=\lfloor(p-1) / 2\rfloor(\lfloor\rfloor$ is the integer division symbol), and $j \operatorname{ssign}\left(\lambda_{k, j+1 / 2}\right)$. The odd and even $\psi$ functions are given by the recurrence formulae (valid for $n \geq 1$ ):

$$
\begin{gathered}
\psi_{k, j+1 / 2}^{2 n}=\sum_{l=0}^{2 n-2}(-1)^{l} \mathbf{C}_{2 n-2}^{l} \cdot\left(c_{2 n} \delta \alpha\right)_{k, j+1 / 2+n-1-l} \\
\psi_{k, j+1 / 2}^{2 n+1}=\sum_{l=0}^{2 n-1}(-1)^{l} \mathbf{C}_{2 n-1}^{l} \cdot\left(c_{2 n+1} \delta \alpha\right)_{k, j+1 / 2+(n-1-l) \cdot j s},
\end{gathered}
$$

where $\mathbf{C}_{r}^{s}=r ! /[(r-s) ! s !]$. The coefficients $c_{q}$ depend on the local CFL number, 
$\nu_{k, j+1 / 2}$, and are given by:

$$
\begin{aligned}
& \left(c_{2}\right)_{k, j+1 / 2}=|\lambda|_{k, j+1 / 2}\left(1-|\nu|_{k, j+1 / 2}\right) \\
& \left(c_{q+1}\right)_{k, j+1 / 2} \frac{|\nu|_{k, j+1 / 2}+(-1)^{q}\lfloor(q+1) / 2\rfloor}{q+1} \cdot\left(c_{q}\right)_{k, j+1 / 2}, \quad q>2
\end{aligned}
$$

This scheme has a stencil of only nine points to get seventh order accuracy in both time and space; it is much less than for multi-stage time integration schemes yielding comparable order of accuracy. The unlimited One-Step highorder scheme (called OSp for the $p$-th order accurate scheme) has a classical CFL stability condition $0 \leq\left|\nu_{k}\right| \leq 1$. Note that this type of scheme yields the exact solution for CFL number equal to 1 , in the scalar case.

TVD-MP constraints: An ad hoc discontinuity-capturing feature must be employed to limit the spurious oscillations in the vicinity of the strong gradient regions. Total Variation Diminishing (TVD) schemes are generally considered to be well suited for the capture of discontinuities. Nevertheless, TVD constraints are known to clip the extrema which appears as a serious drawback in a shock-capturing feature. To avoid this loss of accuracy near extrema, it is necessary to satisfy the Monotonicity-Preserving (MP) criteria introduced by Suresh et al.[20], that enlarge the TVD intervals to provide room for the numerical flux to maintain an accurate value. In Daru et al.[5], we derived a Monotonicity Preserving version of our one-step $p$-th order scheme (named OSMP $p$ ) that preserves accuracy near extrema. In particular, we recast the original MP constraints of Suresh et al.[20] in the TVD framework which allows us to generalize the MP conditions in terms of flux limiting, and avoid any CFL restriction. The MP conditions that preserves accuracy can be expressed directly as constraints on the $\psi^{p}$ function [5].

Let us define the function $\phi^{p}$ such that :

$$
\phi_{k}^{p}=\frac{\psi_{k}^{p}}{\left(1-|\nu|_{k}\right) \delta|f|_{k}}
$$

for each characteristic field. The numerical flux of the OSp scheme can be written, using $\phi^{p}$, as:

$$
F_{j+1 / 2}^{p}=F_{j+1 / 2}^{R o e}+\frac{1}{2} \sum_{k}\left(\phi^{p}(1-|\nu|) \delta|f| \mathbf{r}\right)_{k, j+1 / 2}
$$


If we define :

$$
\begin{aligned}
\phi_{k, j+1 / 2}^{1} & =\frac{2 r_{k, j+1 / 2}}{|\nu|_{k, j+1 / 2}} \cdot \frac{1-|\nu|_{k, j+1 / 2-j s}}{1-|\nu|_{k, j+1 / 2}} \\
\phi_{k, j+1 / 2}^{2} & =\frac{2}{1-|\nu|_{k, j+1 / 2}}
\end{aligned}
$$

the TVD conditions derived by Harten can be expressed as a constraint on $\phi^{p}:$

$$
\phi_{k, j+1 / 2}^{p, T V D}=\max \left\{0, \min \left(\phi_{k, j+1 / 2}^{1}, \phi_{k, j+1 / 2}^{p}, \phi_{k, j+1 / 2}^{2}\right)\right\}
$$

with

$$
r_{k, j+1 / 2}=\frac{\delta \alpha_{k, j+1 / 2-j s}}{\delta \alpha_{k, j+1 / 2}}
$$

The MP constraints are based on the principle that the TVD conditions should be activated only in monotone regions. The following flux variations can be defined :

$$
\left\{\begin{array}{l}
\delta F_{k, j+1 / 2}^{p}=\frac{1}{2} \psi_{k, j+1 / 2}^{p} \\
\delta f_{k, j+1 / 2}^{u l}=\frac{1}{2} \phi_{k, j+1 / 2}^{1} \cdot\left(1-|\nu|_{k, j+1 / 2}\right) \delta|f|_{k, j+1 / 2} \\
\delta f_{k, j+1 / 2}^{m d}=\frac{1}{2} \delta|f|_{k, j+1 / 2}-\frac{1}{2} d_{k, j+1 / 2} \\
\delta f_{k, j+1 / 2}^{l c}=\frac{1}{2} \delta f_{k, j+1 / 2}^{u l}+\frac{1}{2} \frac{1-|\nu|_{k, j+1 / 2-j s}}{|\nu|_{k, j+1 / 2}} d_{k, j+1 / 2-j s}
\end{array}\right.
$$

with $d_{k, j+1 / 2}=\operatorname{minmod}\left(d_{k, j}, d_{k, j+1}\right)$, where $d_{k, j}=(\lambda \delta \alpha)_{k, j+1 / 2}-(\lambda \delta \alpha)_{k, j-1 / 2}$ is a curvature term.

The TVD conditions constrain $\delta F_{k, j+1 / 2}^{p}$ to be inside the interval $\left[0, \delta|f|_{k, j+1 / 2}\right] \bigcap\left[0, \delta f_{k, j+1 / 2}^{u l}\right]$ (this is equivalent to the TVD constraint (16)). The MP conditions provide an enlargement of these intervals : the TVD constraint (16) is activated only when $\delta F_{k, j+1 / 2}^{p}$ is outside the intervals $\left[0, \delta|f|_{k, j+1 / 2}, \delta f_{k, j+1 / 2}^{m d}\right] \bigcap\left[0, \delta f_{k, j+1 / 2}^{u l}, \delta f_{k, j+1 / 2}^{l c}\right]$, where we noted by $\left[f^{1}, f^{2}, \ldots, f^{k}\right]$ the interval $\left[\min \left(f^{1}, f^{2}, \ldots, f^{k}\right), \max \left(f^{1}, f^{2}, \ldots, f^{k}\right)\right]$. In practice, as was done in [20], the criteria is strengthened by choosing the following definition for the curvature measurement $d_{k, j+1 / 2}$ :

$$
d_{k, j+1 / 2}=d_{k, j+1 / 2}^{M 4}=\operatorname{minmod}\left(4 d_{k, j}-d_{k, j+1}, 4 d_{k, j+1}-d_{k, j}, d_{k, j}, d_{k, j+1}\right)
$$

The resulting scheme (OSMP $p$ ) will be $p$ th-order accurate almost everywhere, except around discontinuities where it becomes first order accurate as is the case of all TVD schemes. 
The multidimensional extension is even more delicate than in the case of separate time-space discretization (which is straightforward when treated dimension by dimension [20]), since we have to consider cross derivative terms that appear in the second and higher order terms, which are left uncontrolled if one applies a direction by direction MP correction to a Lax-Wendroff unsplit scheme. A Locally Extremum Diminishing scheme can be obtained if the mixed terms are discretized by using upwind formulae, but is very difficult to implement. The simplest way to avoid this problem of cross derivatives and to recover good properties of the one-dimensional scheme is to use a Strang directional splitting strategy [5], which is only second order accurate. In two dimensions, the system of equations is written:

$$
\frac{\partial w}{\partial t}+\frac{\partial f(w)}{\partial x}+\frac{\partial g(w)}{\partial y}=0
$$

where $f$ and $g$ are the fluxes in each direction. We implement the splitting as follows:

$$
w_{j}^{n+2}=L_{\delta x} L_{\delta y} L_{\delta y} L_{\delta x} w_{j}^{n}
$$

$L_{\delta x}$ (resp. $L_{\delta y}$ ) being a discrete approximation of $L_{x}(w)=-f(w)_{x}$ (resp. $\left.L_{y}(w)=-g(w)_{y}\right)$. In such a way, the second order accuracy is recovered every two time steps.

While reducing the order of accuracy of the original scheme, this simple extension to multidimensional non-linear systems, proposed here, provides a scheme that can compete very well with high order WENO schemes at low cost, as we will see hereafter.

\subsubsection{Method of lines}

Besides the high-order coupled approach, a separate time and space integrations has also been used to solve the governing equations $(1,2)$.

Temporal integration The time integration is performed by means of a third order Runge-Kutta method [18]:

$$
\begin{aligned}
w_{0} & =w^{n} \\
w_{1} & =w_{0}+\delta t \mathcal{L}\left(w_{0}\right) \\
w_{2} & =\frac{3}{4} w_{0}+\frac{1}{4} w_{1}+\frac{1}{4} \delta t \mathcal{L}\left(w_{1}\right) \\
w^{(n+1)} & =\frac{1}{3} w_{0}+\frac{2}{3} w_{2}+\frac{2}{3} \delta t \mathcal{L}\left(w_{2}\right)
\end{aligned}
$$


where $\mathcal{L}(\mathbf{w})$ is a discrete approximation of $\mathcal{L}(\mathbf{w})=-\nabla \cdot\left(\mathbf{f}(\mathbf{w})-\mathbf{f}^{v}(\mathbf{w}, \nabla \mathbf{w})\right)$. This time integration has been chosen since it does not increase the Total Variation of $\mathcal{L}(\mathbf{w})$.

Space discretization The integration of the viscous fluxes is performed by means of a second order centered approximation.

The convective terms are treated following a WENO procedure based on Roe's approximate Riemann solver [15]. The WENO scheme is based on a weighted (convex) combination of the $p$ stencil candidates of the original ENO scheme [16], [17], to increase the order of accuracy of the latter. The weights [9] depend on the degree of regularity of the solution; i.e. in regular regions, the weight coefficients allow one to achieve a $(2 p-1)^{t h}$-order of accuracy while in regions where discontinuities occur, they are set to zero leading to a standard ENO scheme. The WENO fluxes are estimated by:

$$
F_{j+1 / 2}^{W E N O}=\sum_{k}\left[\sum_{l=0}^{p-1} \omega_{l}^{(p)} q_{l}^{p}\left(f_{j+l-p+1}, \ldots, f_{j+l}\right) \mathbf{r}_{k}\right]
$$

where $q_{l}^{p}$ is the ENO reconstruction on the $l^{\text {th }}$ stencil, using the coefficients $\left(\zeta_{l, k}^{p}\right)$ given in Table 1:

$$
q_{l}^{p}\left(f_{j+l-p+1}, \ldots, f_{j+l}\right)=\sum_{k=0}^{p-1} \zeta_{l, k}^{p} f_{k}
$$

and $\omega_{l}^{(p)}$ are the weights defined by:

$$
\begin{aligned}
\sum_{l=0}^{p-1} \omega_{l}^{(p)} & =1 . \\
\omega_{l} & =\frac{\beta_{l}}{\sum_{k=0}^{p-1} \beta_{k}} \\
\beta_{l} & =\frac{C_{l}^{p}}{\left(\varepsilon+I S_{l}\right)^{2}} .
\end{aligned}
$$

$\varepsilon$ is a small positive number to avoid denominator to be zero (hereafter we set $\varepsilon=10^{-6}$ ) and $I S_{l}$ is a regularity measurement of the flux on the $l^{\text {th }}$ stencil candidate. The evaluation of the smoothness measurement $\left(I S_{l}\right)$ is based on the undivided-differences [9].

Hereafter, the calculations were performed by using $p=5$ that gives a theoretical $9^{\text {th }}$-order of accuracy in space. The corresponding ENO coefficients are 
Table 1

\begin{tabular}{||c||c||c|c|c|c|c||}
\hline \hline$p$ & $l$ & $k=0$ & $k=1$ & $k=2$ & $k=3$ & $k=4$ \\
\hline \hline 5 & 4 & $1 / 5$ & $-21 / 20$ & $137 / 60$ & $-163 / 60$ & $137 / 60$ \\
\hline & 3 & $-1 / 20$ & $17 / 60$ & $-43 / 60$ & $77 / 60$ & $1 / 5$ \\
\hline & 2 & $1 / 30$ & $-13 / 60$ & $47 / 60$ & $9 / 20$ & $-1 / 20$ \\
\hline & 1 & $-1 / 20$ & $9 / 20$ & $47 / 60$ & $-13 / 60$ & $1 / 30$ \\
\hline & 0 & $1 / 5$ & $77 / 60$ & $-43 / 60$ & $17 / 60$ & $-1 / 20$ \\
\hline & -1 & $137 / 60$ & $-163 / 60$ & $137 / 60$ & $-21 / 20$ & $1 / 5$ \\
\hline
\end{tabular}

ENO coefficients $\left(\zeta_{l, k}^{p}\right)$ for $\mathrm{p}=5$ on a regular mesh.

\begin{tabular}{||c||c|c|c|c|c||}
\hline \hline$\omega_{l}^{(p)}$ & $l=0$ & $l=1$ & $l=2$ & $l=3$ & $l=4$ \\
\hline$p=5$ & $1 / 126$ & $10 / 63$ & $10 / 21$ & $20 / 63$ & $5 / 126$ \\
\hline \hline
\end{tabular}

Table 2

Weights $\left(\omega_{l}^{(p)}\right)$ for the WENO $(\mathrm{p}=5)$ procedure

reported in Table (1) while the weights are given in Table (2).

\subsection{Boundary conditions}

On the solid wall boundaries, a no-slip condition is prescribed on the velocity components. The density is calculated through the use of the continuity equation written on the wall points. An adiabatic condition is prescribed on the wall temperature. A standard fourth order upwind formula is used to estimate the normal to the wall derivatives.

\section{Flow field configuration}

We consider a square shock tube with a unit side length and insulated walls. The diaphragm is initially located at the middle of the tube $(x=0.5)$. The initial states, in terms of dimensionless quantities, are on the left-side of the diaphragm: $\rho_{L}=120, P_{L}=\rho_{L} / \gamma, u_{L}=v_{L}=0$, and on the right-side: $\rho_{R}=1.2$, $P_{R}=\rho_{R} / \gamma, u_{R}=v_{R}=0$. The reference velocity is based on the initial speed of sound, corresponding to a reference Mach number $M_{0}=1$. The Prandtl number is assumed constant ( $\mathrm{Pr}=0.73$, for air). Four Reynolds numbers have been considered: $R e=200,500,750,1000$. At the initial time, the diaphragm is broken. The 1-D inviscid solution is presented in Fig. 1, showing the evolution of the density (left) and the velocity (right) in the $x-t$ plane. A shock 
wave, followed by a contact discontinuity, moves toward the low-pressure region (i.e. to the right, in the present case), while a sonic rarefaction wave moves toward the high-pressure region at the left. The shock Mach number is equal to 2.37. The rarefaction wave reflects on the left wall at a dimensionless time $t=0.5$. The incident shock wave is weak, and reflects at the right wall at time $t \approx 0.21$. After reflection, it interacts with the contact discontinuity. Complex interactions then occur leading to transmitted and reflected waves as one can see in figure 1. For later times, the contact discontinuity stays stationary, close to the right wall. On the contrary, the reflected shock wave moves on and begins to interact with the rarefaction wave at time $t=0.4$, and later with the reflected rarefaction wave. During the interaction the reflected shock has an approximately constant velocity, but the fluid velocity decreases continuously after its interaction with the incident rarefaction wave (Fig. 1 right).
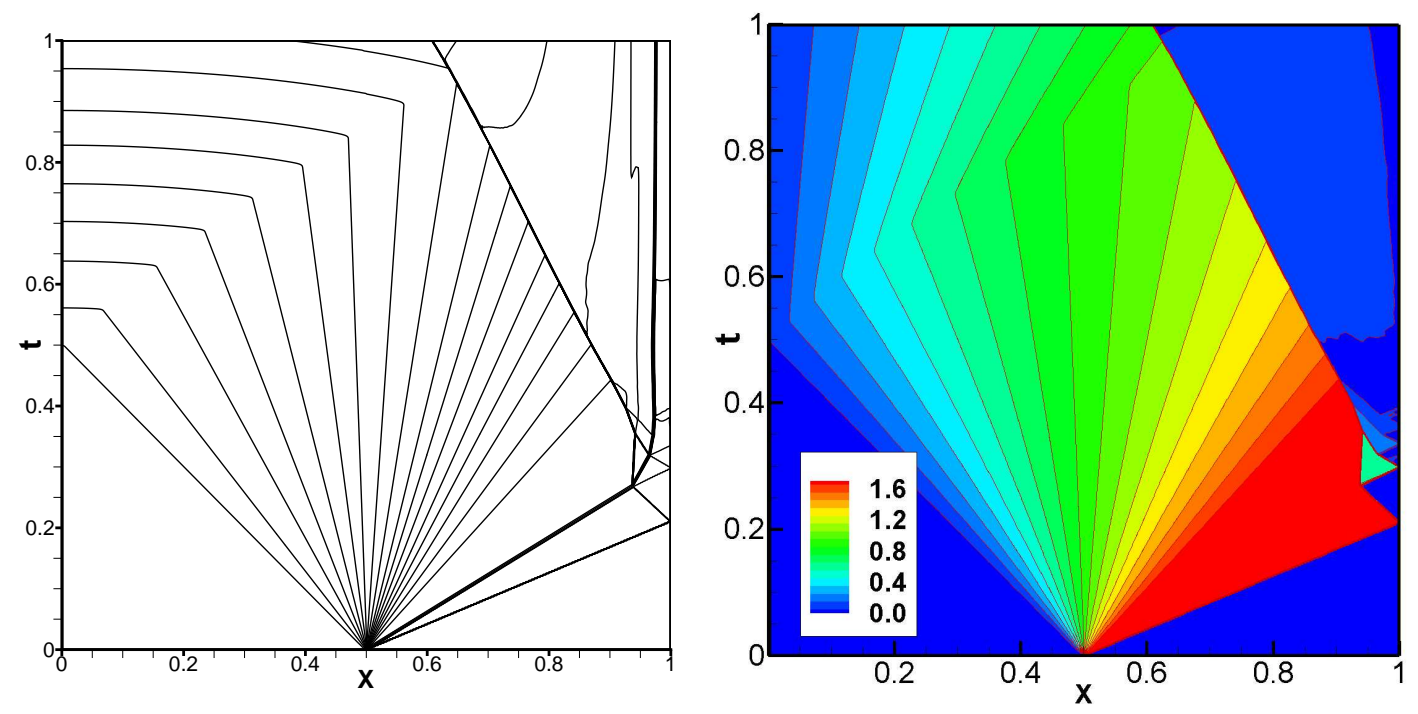

Fig. 1. $x-t$ diagram of the density (left), velocity (right).

In the $2 \mathrm{D}$ viscous case, the propagating incident shock wave and contact discontinuity interact with the horizontal wall, creating a thin boundary layer. After its reflection on the right wall, the shock wave interacts with this boundary layer. As the stagnation pressure in the boundary layer is lower than the one within the outflow region, a separation region (named "bubble") appears over a large extent within the boundary layer, resulting in a major modification of the flow pattern and the formation of "a lambda-shape like shock pattern", as one can see in figure 6 . The triple point emerging from the lambda-shape like shock pattern generates a slip line that rolls up in the right end corner. 


\section{Grid convergence study and influence of the schemes}

The simulations have been carried out for four Reynolds numbers: $R e=$ 200, 500, 750 and 1000. Simulations with increasing grid resolutions have been performed to verify grid-independency of the solutions. All meshes use equally spaced points with cell aspect ratio equal to unity. The grid convergence is shown for the density distribution along the bottom wall at $t=1$ (Fig. 2). To make sure that other quantities are well converged, we also provide the

(a)

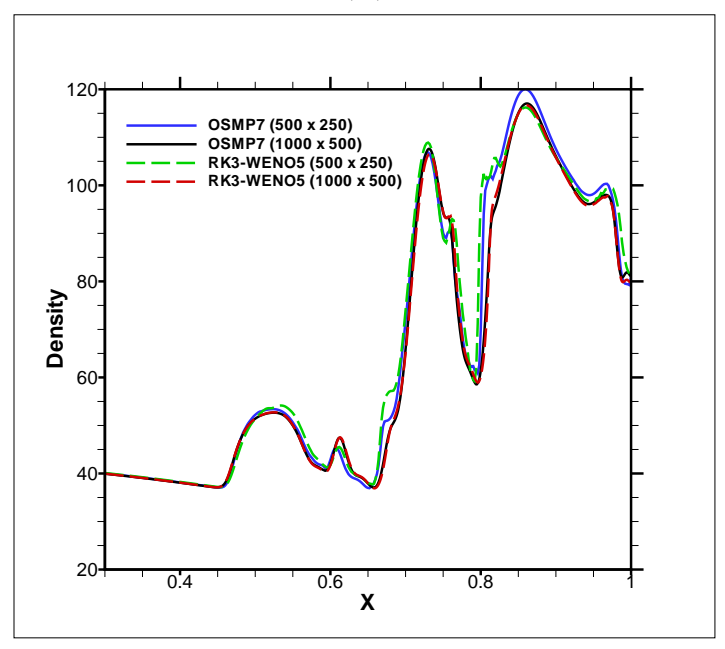

(c)

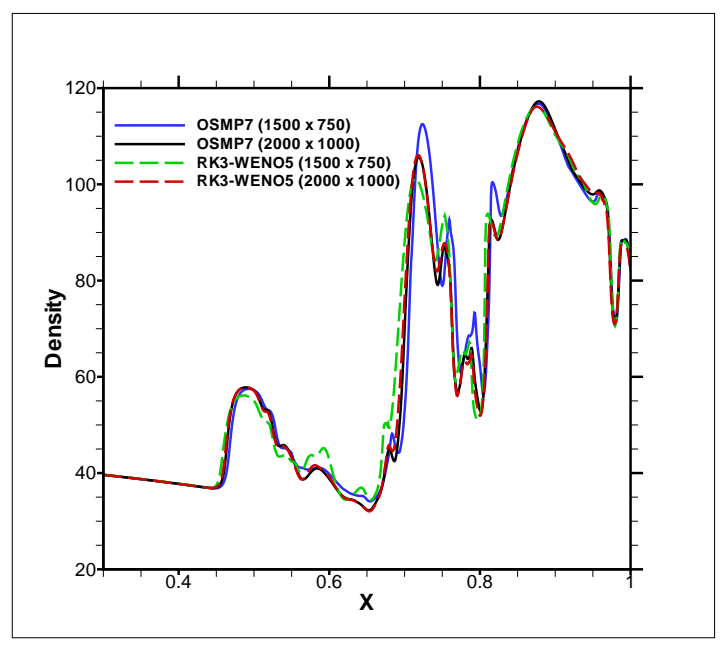

(b)

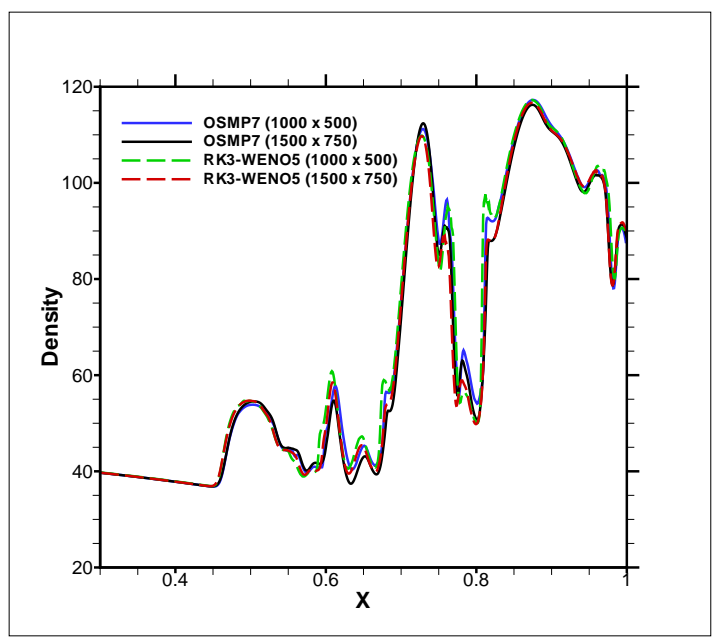

(d)

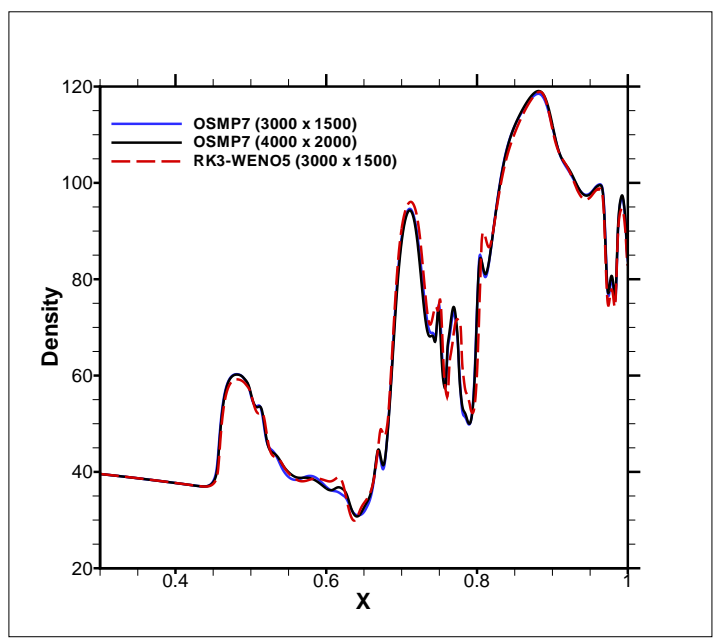

Fig. 2. Density distributions along the bottom wall of the shock tube, obtained at $t=1$ on several fine grids by using the OSMP7 scheme (solid lines) and the RK3-WENO5 scheme (dashed lines), for four Reynolds numbers: (a) $R e=200$, (b) $R e=500,(\mathrm{c}) R e=750$ and (d) $R e=1000$.

distributions of the skin friction coefficient along the bottom-wall for the four Reynolds numbers (Fig. 3). Furthermore, for $R e=1000$, we plot the den- 
(a)

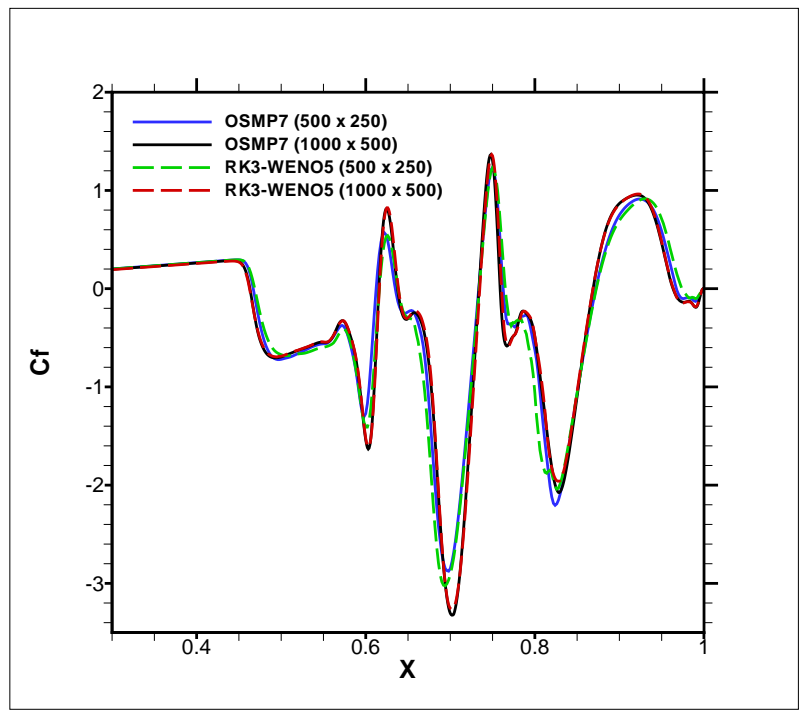

(c)

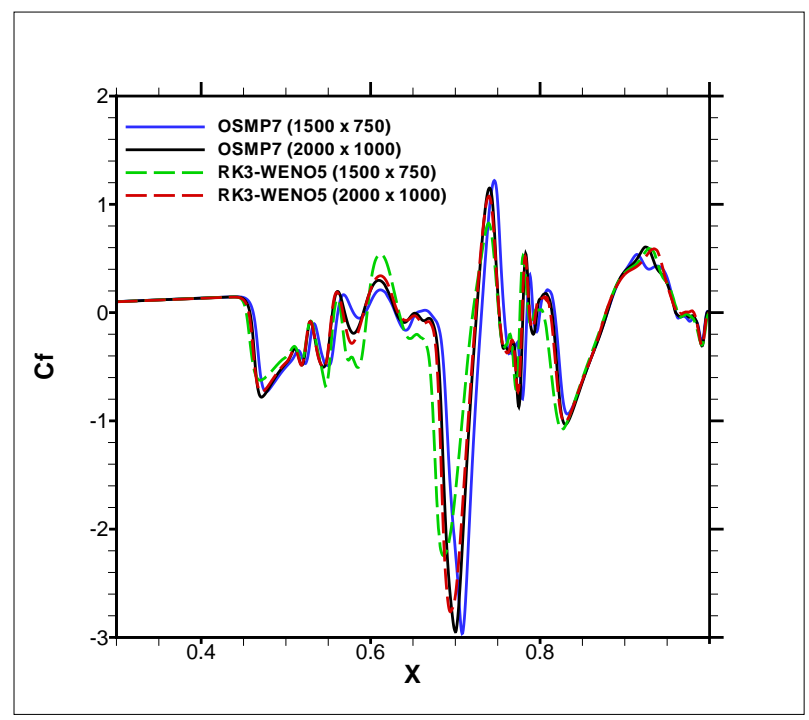

(b)

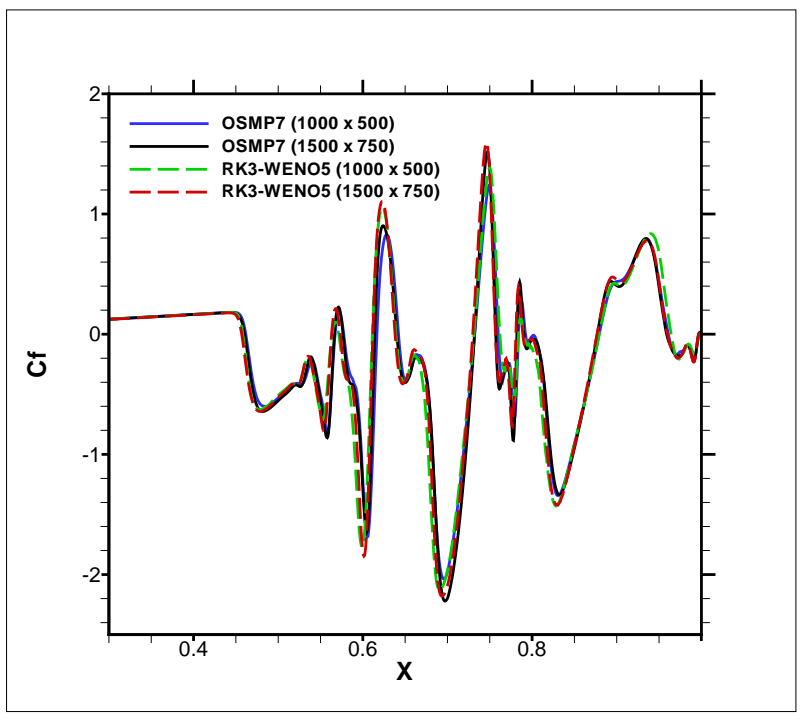

(d)

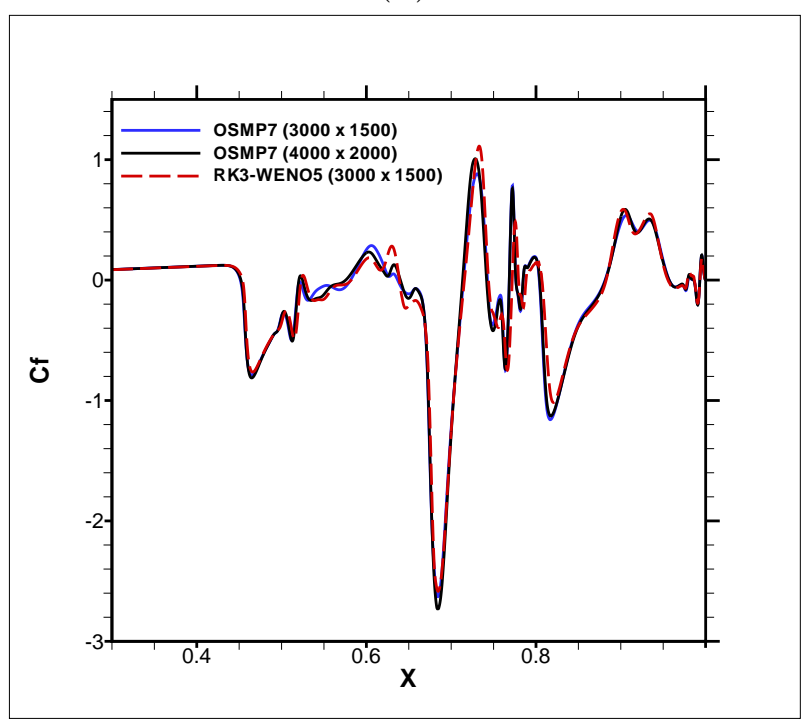

Fig. 3. Distributions of the skin friction coefficient along the bottom wall of the shock tube, obtained at $t=1$ on several fine grids by using the OSMP7 scheme (solid lines) and the RK3-WENO5 scheme (dashed lines), for four Reynolds numbers: (a) $R e=200,(\mathrm{~b}) R e=500,(\mathrm{c}) R e=750$ and (d) $R e=1000$.

sity (Fig. 4-a) and the vorticity (Fig. 4-b) along the horizontal line crossing the separation bubble and the vortices, located at $y=0.05$. Simulations for RK3-WENO5 scheme on the finest grid $(4000 \times 2000)$ at $R e=1000$ were not performed due to prohibitive computational cost.

As expected, greater Reynolds numbers require finer grids to achieve convergence. From this numerical experiment, we found that sufficient grid resolutions for the different Reynolds numbers are: $1000 \times 500$ for $R e=200$ (Fig. 2-a 
(a)

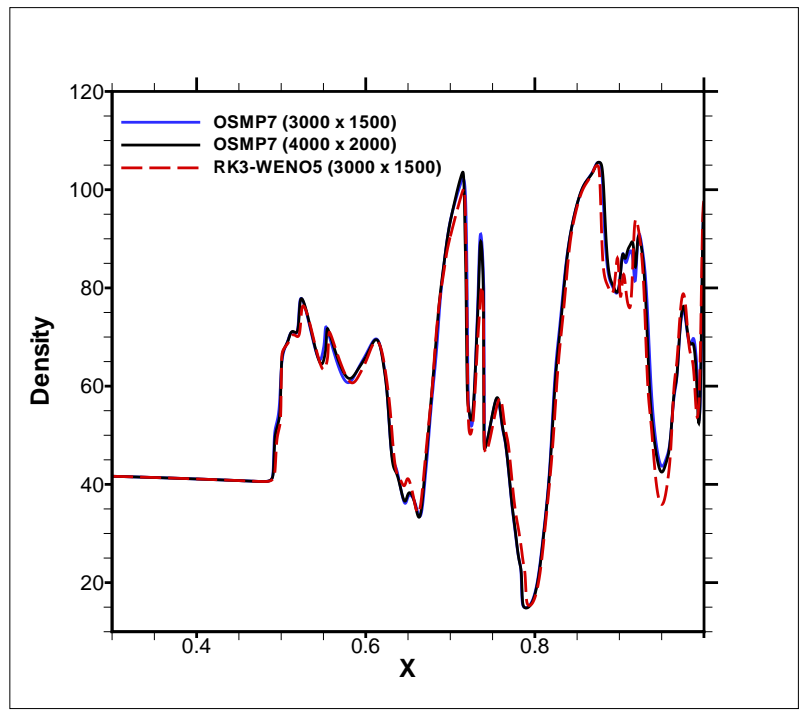

(b)

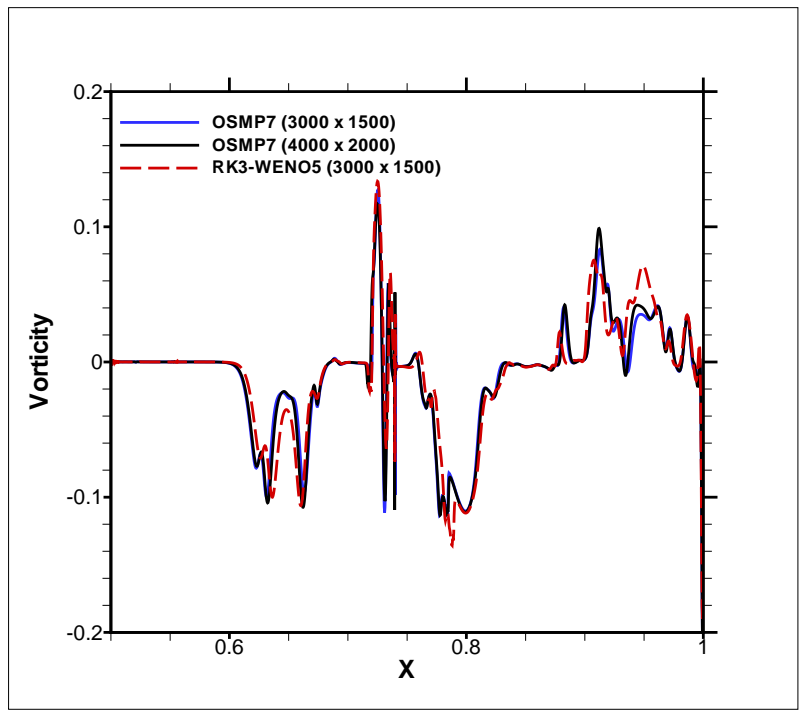

Fig. 4. Distributions of density (a) and the vorticity (b) along the line $y=0.05$, obtained at $t=1$ on several fine grids by using the OSMP7 scheme (solid lines) and the RK3-WENO5 scheme (dashed lines), for $R e=1000$.

and 3-a), $1500 \times 750$ for $R e=500$ (Fig. 2 -b and 3-b), $2000 \times 1000$ for $R e=750$ (Fig. 2-c and 3-c) and $4000 \times 2000$ for $R e=1000$ (Fig. 2-d, 3-d and 4).

In a previous study [3], we have shown convergence issues when using classical shock capturing TVD approaches for this test-case, especially at $R e=1000$. Subsequently, Sjögreen and Yee [19] have unsuccessfully attempted to obtain converged solutions by using several separate time and space schemes (MUSCL-RK2, WENO5-RK4 and high order filtered difference methods). For instance, the solution for high order filtered difference methods is notably different than the solution for MUSCL-RK2 method. Also, for $R e=1000$, they obtained solutions significantly different than ours. They mentioned that one cannot conclude on the respective accuracy of the various schemes and that the stability of the flow is questionable. In our simulations, converge solutions are clearly obtained as seen in previous figures and also in figure 5 where plotted are the density fields at $t=1$ for several grids. Again, results for RK3-WENO5 scheme on the finest grid $(4000 \times 2000)$ are lacking in figure 5 because of the prohibitively large CPU time required, but it is obvious that the two numerical methods tend to converge to the same solution.

Computations were performed on a NEC-SX8, at the CNRS's National Computing Center (IDRIS). The OSMP7 scheme takes about $3.10^{-7}$ s.CPU per time step and grid point, at a flow-rate of 7 Giga Flops. The CPU time required by the RK3-WENO5, at a flow-rate of 6 Giga Flops, is $2.10^{-6}$ s.CPU per time step and grid point ie six to seven times greater than the OSMP7 scheme. 
(a)

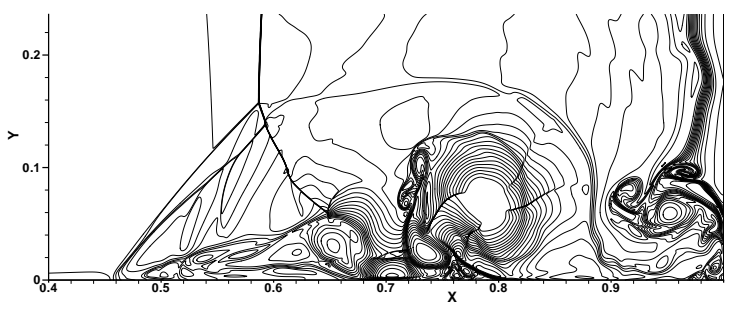

(b)

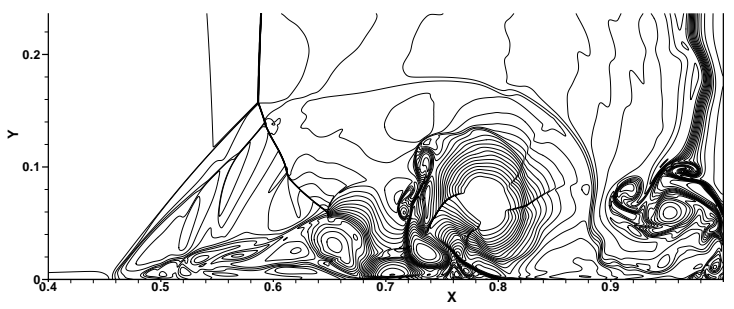

(d)

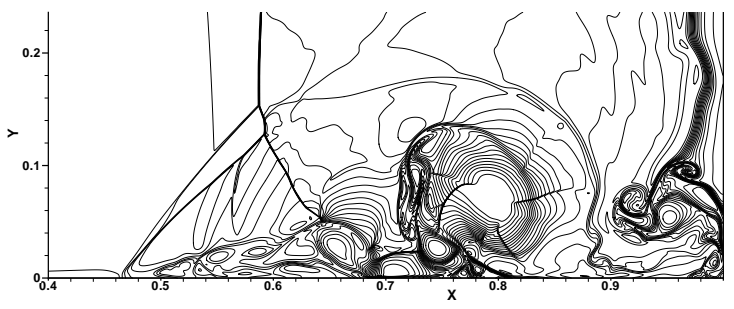

(c)

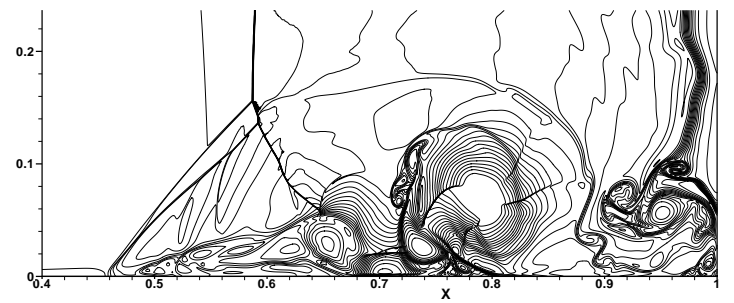

(e)

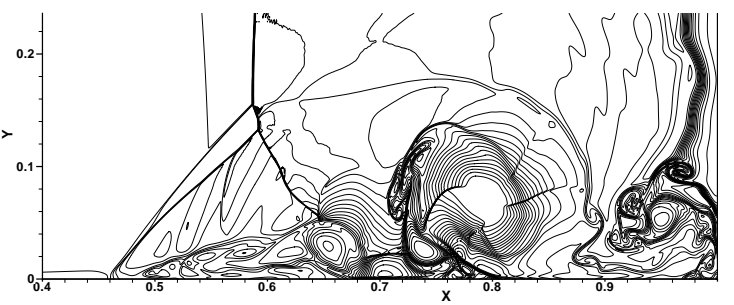

Fig. 5. Contours of $\rho$ obtained on the finest grid at $t=1$ for $R e=1000$ : (a) OSMP7 $(4000 \times 2000) ;($ b) RK3-WENO5 $(3000 \times 1500) ;($ c) OSMP7 $(3000 \times 1500) ;(d)$ RK3-WENO5 $(2000 \times 1000)$; (e) OSMP7 $(2000 \times 1000)$. $(21$ contour levels between 20 and 120).

We can observe that the OSMP7 scheme exhibits a faster grid convergence than the RK3-WENO5 scheme on the vortical structure in the bottom-right corner due to the roll-up of the contact discontinuity. This can mainly be attributed to the poor behavior of the WENO scheme for contact discontinuities. However, the convergence of the main vortical structure (downstream of the lambda-like shock pattern) seems to be rather identical for both schemes. Though very small differences are seen between the $3000 \times 1500$ and $4000 \times 2000$ mesh solutions, we can consider that the solutions on the $3000 \times 1500$ grid are almost converged for the two schemes. This support the claim of a converged solution for the $4000 \times 2000$ mesh and OSMP7 scheme, for the highest considered Reynolds number $(R e=1000)$. We suggest that our converged solutions could be used as a reference in future work. 


\section{Dynamic flow analysis of a shock wave / boundary layer inter- action.}

This section is devoted to the analysis of the flow dynamics during the interaction of the reflected shock-wave with the laminar boundary layer. The interaction begins at the bottom-right corner right after the incident shock wave has reflected on the right wall. When the reflected shock interacts with the bottom wall boundary layer, the stagnation pressure within the boundary layer is lower than downstream of the normal shock. After the shock reflection, the hotter fluid in contact with the right wall moves suddenly down to the bottom wall, creating a hot countercurrent jet close in the corner. A recirculating flow is then generated that initiates the boundary layer detachment to form a "bubble". The deviation of the cold flow over the slip line (supersonic shear layer) delimiting the hot fluid "bubble" then induces a lambda-shape like shock pattern. This early stage of the reflected shock/boundary interaction can be seen at a dimensionless time $t=0.4$, on figure (6) where the static temperature contours are plotted for the solution at $R e=200$ obtained on the finest grid. A short time later, the shock interacts with the incident contact discontinuity. The incident flow in between the lambda-shock and the wall jet, rolls up around the bubble and forms a high-speed cold jet. After this jet has impinged on the bottom wall (around $t=0.5$ as we can see in figure (6)), no more hot shocked fluid enters the bubble, which is isolated from the hot right wall region. The countercurrent part of the jet then alternatively hits and reflects between the bottom wall and the supersonic shear layer originating from the separation point (Fig. 6), thus creating a series of counter-rotating vortices. Each vortex is made up of three "layers" of fluid : at the center the hottest shocked fluid, next a layer of colder fluid originating from the boundary layer, and finally the coldest layer of fluid from the external flow at the periphery (see Fig. 6). The jet flow is very energetic with supersonic velocities at several places. Each impact of the jet, either on the solid wall or on the oblique slip line, creates a relatively high pressure region. This leads to the deformation of the slip line and to the generation of secondary shock waves above it.

\section{Influence of Reynolds number on the flow dynamics.}

The analysis of the flow dynamics within the separation region has pointed out that the speed of the counter flow jet increases with the Reynolds number. Consequently, during a fixed period of time, the number of jet alternated reflections between the bottom wall and the slip line increases with Re. Also, the number of counter rotating vortices produced in this interaction region increases too. This can be seen from the density gradient and temperature 
(a)

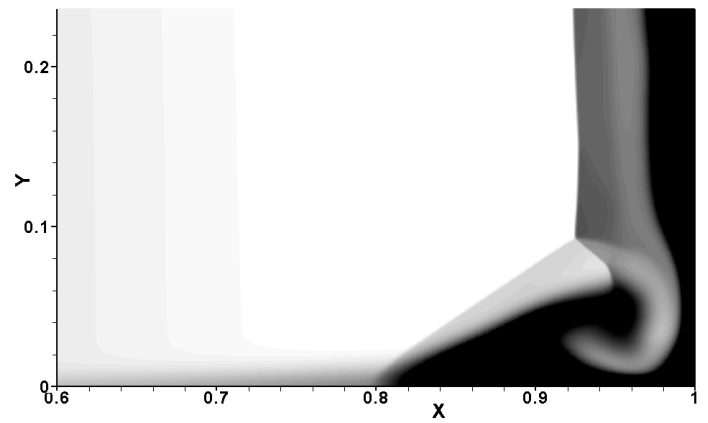

(c)

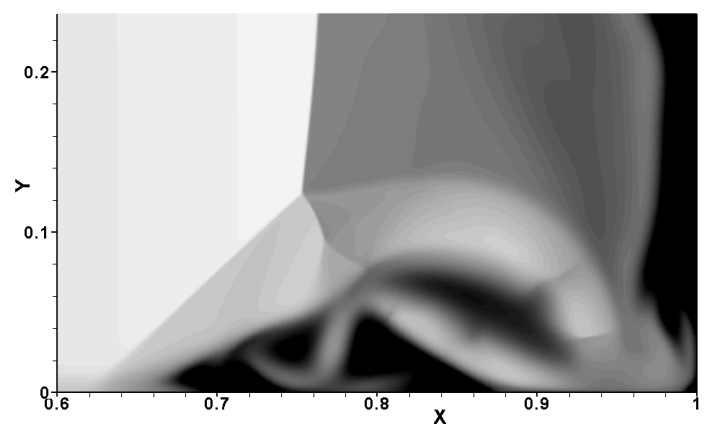

(b)

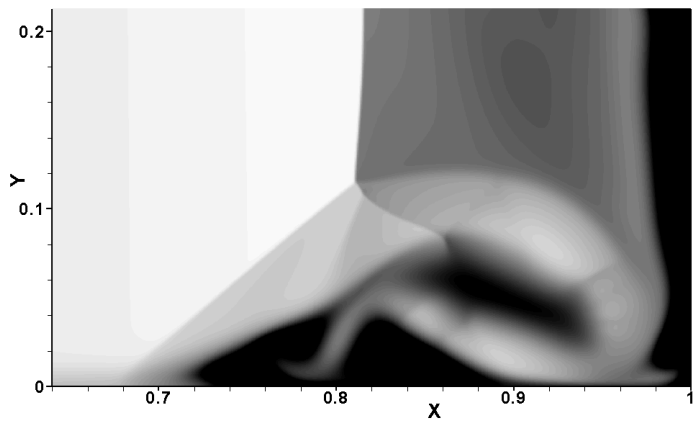

(d)

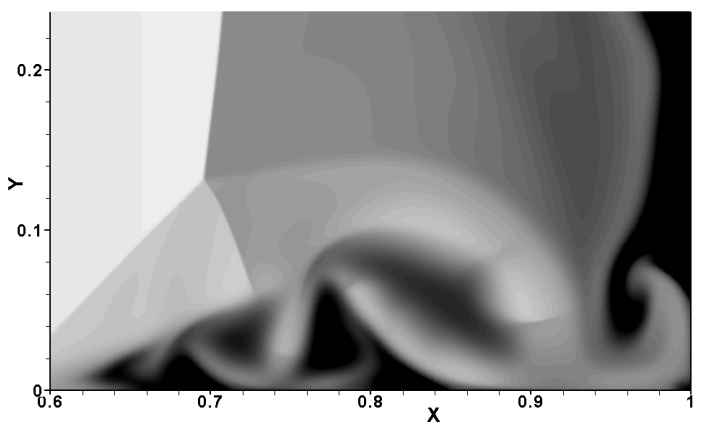

Fig. 6. Contours of $T$ obtained on the finest grid for $R e=200$ at (a) $t=0.4$, (b) $t=0.6$, (c) $t=0.7$, (d) $t=0.8$. Hot fluid in dark, cold fluid in light. 41 niveaux entre 0.4 et 1.2

fields in Fig. 7 and 8, where the solutions for the four Reynolds numbers are presented at $t=0.6$ and $t=1$ respectively. Shocklets in the vicinity of the vortices as well as Kelvin-Helmholtz instabilities in the supersonic shear layer originating at the triple point are visible on the contours of density gradients (Fig. 7-left and 8-left). The temperature fields (on Fig. 7-right and 8-right) give additional information on the flow, notably on the location of hot and cold streams and the flow coming from the initial boundary layer.

The Reynolds number also affects the location of the triple point of the lambda-shape like shock pattern. One can observe, in figures 7 and 8, that the height of the triple point increases with $R e$. To better appreciate the motion of the triple point, its coordinates are plotted as a function of time in Fig. 9 for the four Reynolds numbers. Its horizontal velocity is constant and nearly independent of $R e$ (Fig. 9-left). This velocity is slightly greater than that of the normal shock along the tube centerline $(y=0.5)$. On the contrary, the triple point motion along the vertical direction depends on the Reynolds number (Fig. 9-right). Time evolutions of the triple point distance to the bottom wall, are in agreement with theoretical predictions (e.g. see [7], [13] and [14]). Similarly to Matsuo et al. [14], we found two periods before and after $t=0.53$ where the $R e$ dependency changes. Surprisingly, at $t=0.53$ all the 
curves cross each other. In the first period (before $t=0.53$ ), the triple point height is lower for higher Re due to the thinner boundary layer. We found that $t=0.53$ corresponds to the time when the bubble is "closed", i.e. when the heated vertical jet, coming from the fluid flowing in between the lambda-shock and rolling up around the bubble, impinges on the bottom wall and separates the right wall hot region from the bubble. We have no explanation to propose for the $R e$ independency of the closing time of the bubble. Anyway, before $t=0.53$, the separated bubble is fed by both the incoming boundary layer and the heated vertical jet. On the contrary, after that time, the detachment is only fed by a portion of the boundary layer, the bubble being "closed". In accordance with [7], [13] and [14], we verified in our results that the pressure downstream the leading oblique shock wave (under the lambda-shock) is the same than the pressure at the beginning of the detachment which is equal to the stagnation pressure in the incoming boundary layer. In a crude theory, Mark [13] showed that the stagnation pressure in the boundary layer is only related to the Mach number of the reflected shock wave and is independent of the Reynolds number. In the flow condition of our test-case, Marks's theory was corroborated by more sophisticated developments undertaken by Davies et al. [7] and Matsuo et al. [14]. The ratio between the boundary layer stagnation pressure and the static pressure (above the boundary layer) upstream of the leading reflected shock can be determined using the Mach number of the reflected shock wave [13] (in the present case $M_{s}=2.37$ ). This Mach number being independent of $R e$, it explains the constant angle of the leading oblique shock wave for all $R e$ found in the simulations. However, the velocity of the jet increases with Re leading to increasing bubble size, as predicted by Mark [13]. Since the angle of the leading oblique shock is constant, it is shifted to the left with increasing distance from the bottom wall of the triple point for increasing Re (Fig. 9-right).

The Reynolds number effects also impacts the vortices created within the lambda shock pattern, i.e. at $t=0.6$ (Fig. 7): three vortices are created at $R e=200$ and six at $R e=1000$. When the number of vortices increases, additional shocks are created inside the lambda shock pattern. To further analyze the dynamical process, we have also plotted, in a coordinate reference frame attached to the reflected normal shock, the vorticity field with some streamlines superimposed. These plots are presented at several dimensionless times and for the four Re studied (Fig. 10, 11, 12, 13). For the lowest Reynolds numbers, $R e=200$ and $R e=500$, Fig. 10 and 11 show a regular arrangement of the vortices inside the bubble. This regular arrangement disappears for $R e \geq 750$ (Fig. 12 and 13). Detailed examination of the dynamics of the flow reveals the following process : eddy structures are created close to the bottom wall, with an increasing enstrophy for increasing Re (Fig. 14). Similar to the vortex/boundary layer interaction, when the enstrophy of the eddy structures is large enough, they are ejected out of the boundary layer. Hence, the structures (mainly the second one from the right as indicated on figures 12-b and 
(a) $R e=200$
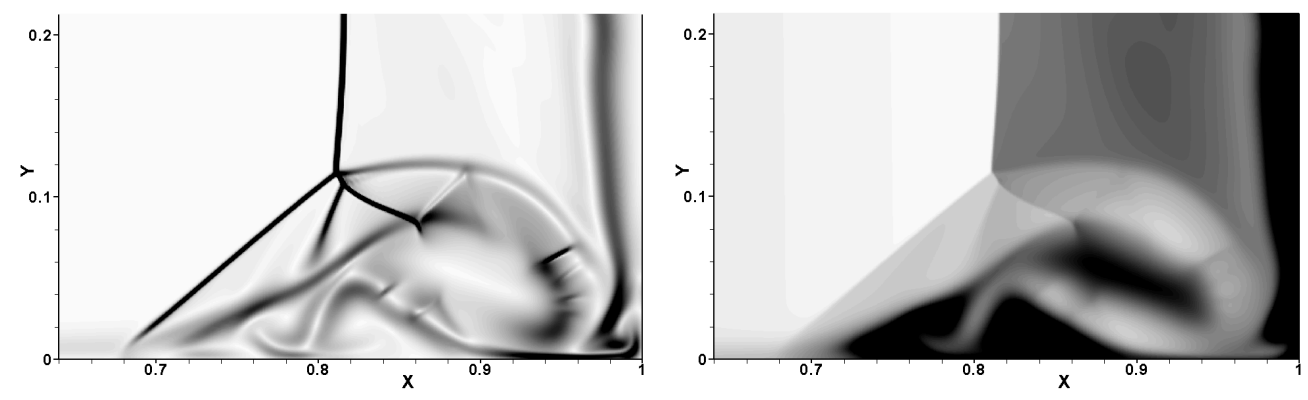

(b) $R e=500$
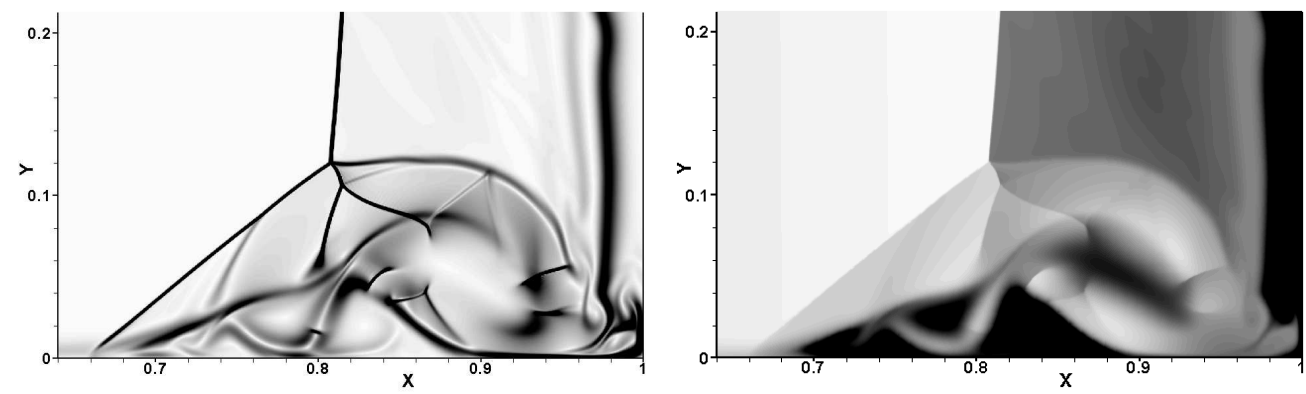

(c) $R e=750$
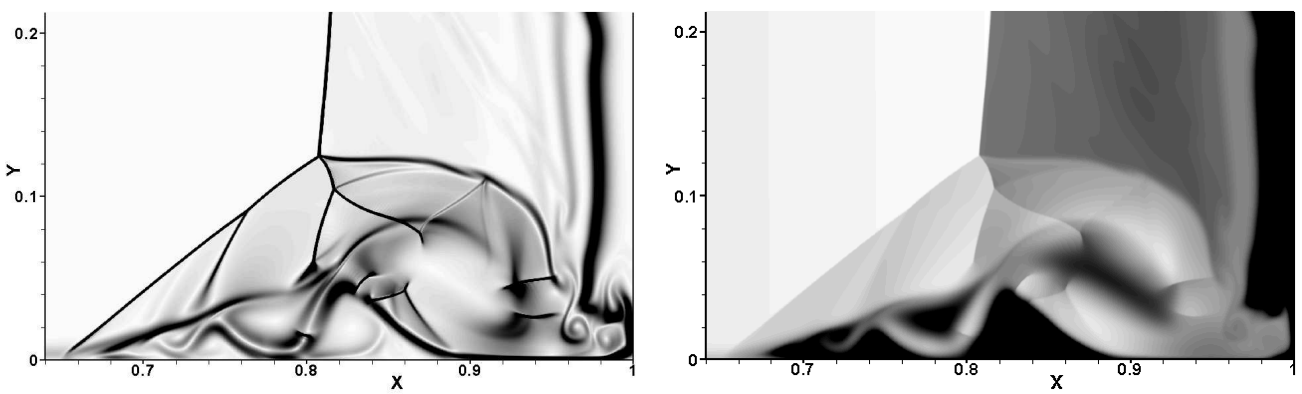

(d) $R e=1000$
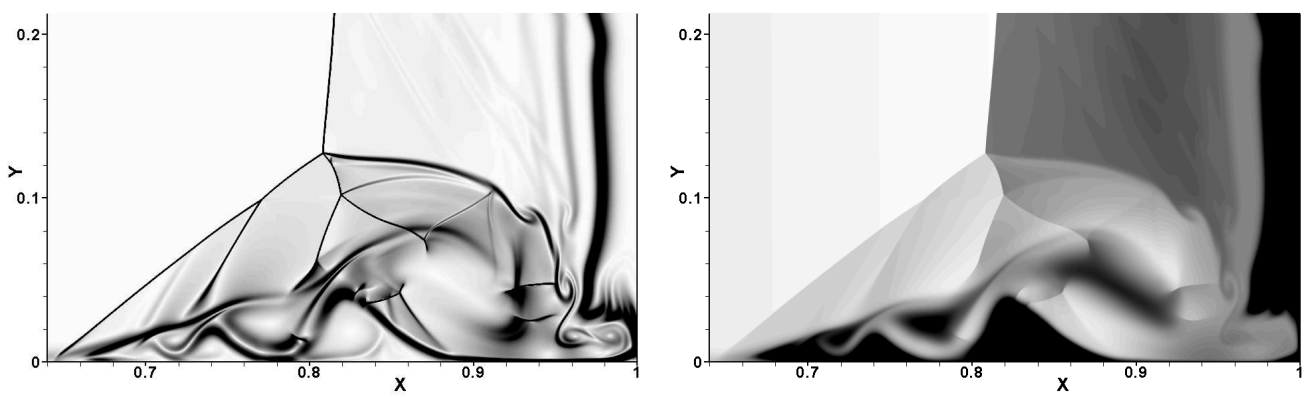

Fig. 7. Contours of $|\nabla \rho|$ (left) and $T$ (right, 41 levels entre 0.4 et 1.2) obtained at $t=0.6$ on the finest grid for several Reynolds numbers: (a) $R e=200$; (b) $R e=500$; (c) $\operatorname{Re} 750 ;$ (d) $R e=1000$.

13-b) are stretched and distorted into an intense vortex filament (shown on figures 12-b and 13-b). This filament is then ejected out of the boundary layer and trapped, through a pairing process, with the largest vortical structure (especially, the first structure from the right, as indicated on figures 12-b and 
(a) $R e=200$
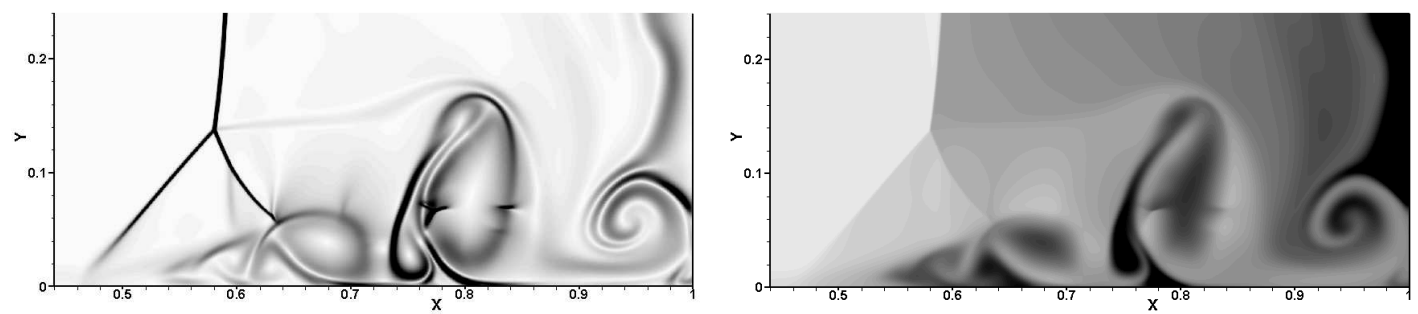

(b) $R e=500$
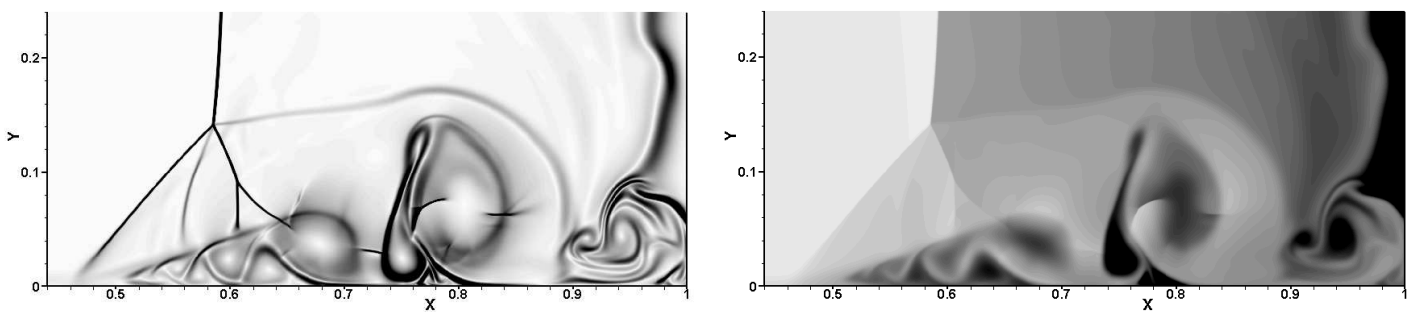

(c) $R e=750$
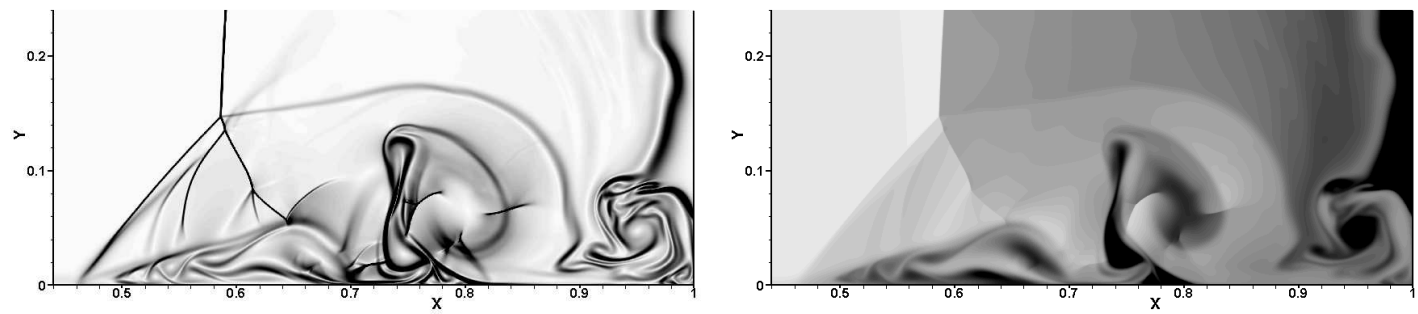

(d) $R e=1000$
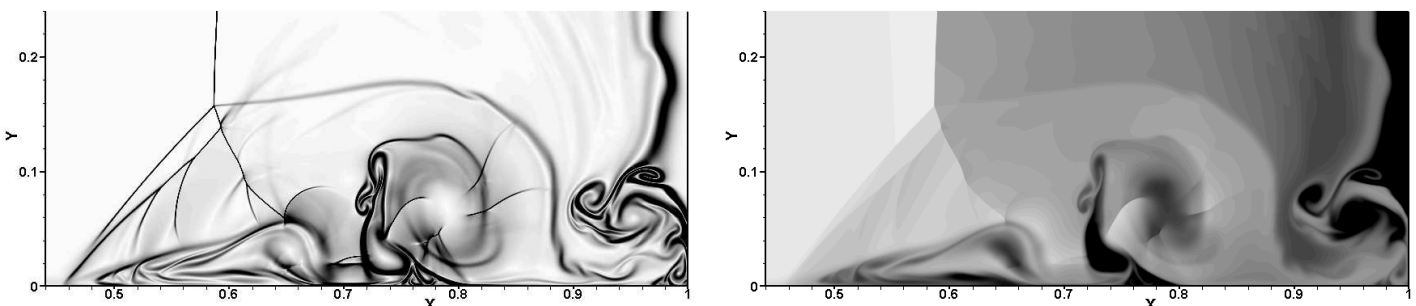

Fig. 8. Contours of $|\nabla \rho|$ (left) and $T$ (right, 41 levels entre 0.4 et 1.2) obtained at $t=1$ on the finest grid for several Reynolds numbers: (a) $R e=200$; (b) $R e=500$; (c) $\operatorname{Re} 750 ;$ (d) $R e=1000$.

13-b). Due to the shear stress of the outflow, the filament is elongated to the point where it breaks up. After that, the enstrophy intensity decreases close to the wall, resulting in the retraction of the structure with a "spring back effect". This in turn affects the vortex arrangement which becomes irregular, with flattened structures. Neighboring co-rotating structures eventually merge. 

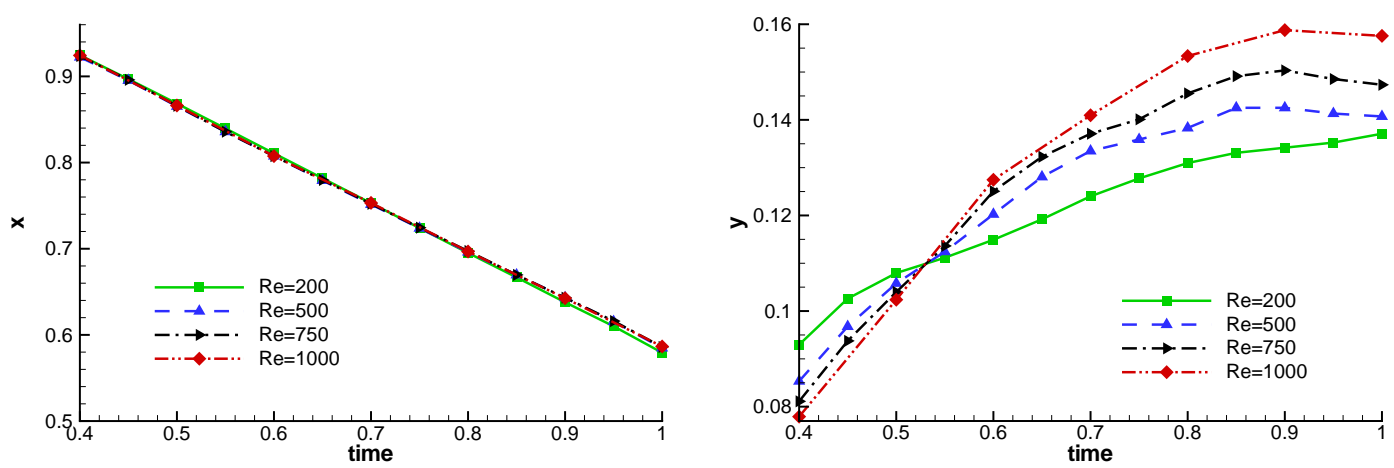

Fig. 9. $\mathrm{x}$ (left) and y (right) coordinates of the triple point versus time

\section{Conclusion}

This study demonstrates that the calculation of such a complex flow requires numerical schemes which are both robust and very accurate. The OSMP7 scheme allows to reach fully converged solutions for Reynolds numbers as high as 1000. Simulations with sufficiently refined grids to obtain converged solutions could not be performed using the classical WENO scheme because of the excessive CPU cost. Using converged solutions, the flow dynamics and its evolution with the Reynolds number were analyzed in detail. The present analysis is only valid for 2-D laminar configurations. For larger $R e, 3-\mathrm{D}$ instabilities will change the present flow dynamics. Nevertheless, by considering the $R e$ range we studied, this $2 \mathrm{D}$ shock tube problem is an appropriate test-case for validation of high-resolution schemes, and the presented grid-converged results may serve as reference solutions for that purpose.

Acknowledgements: Computations have been carried out on NEC-SX8 of the CNRS's national supercomputing center (IDRIS). The authors greatly acknowledge the support of this institution.

\section{References}

[1] Y. Burtschell, M. Cardoso, D. Zeitoun, Numerical analysis of reducing driver gas contamination in impulse shock tunnels., AIAA Journal 39 (12) (2001) 2357-2365.

[2] V. Daru, X. Gloerfelt, Aeroacoustic computations using a high-order shock-capturing scheme, AIAA Journal 45 (2007) 2474-2486.

[3] V. Daru, C. Tenaud, Evaluation of tvd high resolution schemes for unsteady viscous shocked flows, Computers \& Fluids 30 (2001) 89-113.

[4] V. Daru, C. Tenaud, High resolution monotonicity preserving schemes for unsteady compressible flows., in: K. S. E. S. Armfield, P. Morgan (ed.), 
Proceedings of the ICCFD'2 Conference, July 2002, Sydney, Australia., Springer-Verlag, 2002.

[5] V. Daru, C. Tenaud, High order one-step monotonicity preserving schemes for unsteady flow calculations, Journal of Computational Physics 193 (2004) 563-594.

[6] V. Daru, C. Tenaud, Numerical simulation of the shock wave / boundary layer interaction in a shock tube by using a high resolution monotonicitypreserving scheme., in: Proceedings of the ICCFD'3 Conference, July 2004, Toronto, Canada., 2004.

[7] L. Davies, J. Wilson, Influence of reflected shock and boundary-layer interaction on shock-tube flows., in: Proceedings of the Shock Tube Symposium, The Physics of Fluids Supplement I., 1969.

[8] R. Goozée, P. Jacobs, D. Buttswortth, Simulation of a complete reflected shock tunnel showing a vortex mechanism for flow contamination., Shock Waves 15 ((3-4)) (2006) 165-176.

[9] G.-S. Jiang, C.-W. Shu, Efficient implementation of weighted eno schemes, Journal of Computational Physics 126 (1996) 202-228.

[10] K. Kim, C. Kim, Accurate, efficient and monotonic numerical methods for multi-dimensional compressible flows: Part i: Spatial discretization., Journal of Computational Physics 208 (Issue 2) (2005) 527-569.

[11] K. Kim, C. Kim, Accurate, efficient and monotonic numerical methods for multi-dimensional compressible flows: Part ii: Multi-dimensional limiting process., Journal of Computational Physics 208 (Issue 2) (2005) 570-615.

[12] D. Lax, B. Wendroff, Systems of conservation laws, Communications on Pure and Applied Mathematics 13 (1960) 217-237.

[13] H. Mark, The interaction of a reflected shock wave with the boundary layer in a shock tube., Technical Memorandum 1318, NASA (1958).

[14] K. Matsuo, S. Kawagoe, K. Kage, The interaction of a reflected shock wave with the boundary layer in a shock tube., Bulletin of the ASME 17 (110) (1974) 1039-1046.

[15] P. L. Roe, Approximate riemann solvers, parameter vectors, and difference schemes, Journal of Computational Physics 43 (1981) 357-372.

[16] C. W. Shu, S. Osher, Efficient implementation of essentially nonoscillatory shock-capturing schemes, Journal of Computational Physics 77 (1988) 439-471.

[17] C. W. Shu, S. Osher, Efficient implementation of essentially nonoscillatory shock-capturing schemes, II, Journal of Computational Physics 83 (1989) 32-78.

[18] C. W. Shu, T. A. Zang, G. Erlebacher, D. Whitaker, S. Osher, High order eno schemes applied to two and three dimensional compressible flows, Applied Numerical Mathematics Transactions of IMACS 9 (1992) 45-71.

[19] B. Sjogreen, H. C. Yee, Grid convergence of high order methods for multiscale complex unsteady viscous compressible flows, Journal of Computational Physics 185 (2003) 1-26. 
[20] A. Suresh, H. T. Huynh, Accurate monotonicity-preserving schemes with runge-kutta time stepping, Journal of Computational Physics 136 (1997) 83-99.

[21] Y. S. Weber, E. S. Oran, J. P. Boris, J. D. Anderson, The numerical simulation of shock bifurcation near the end wall of a shock tube, Physics of Fluids 7 (10) (1995) 2475-2488. 
(a)

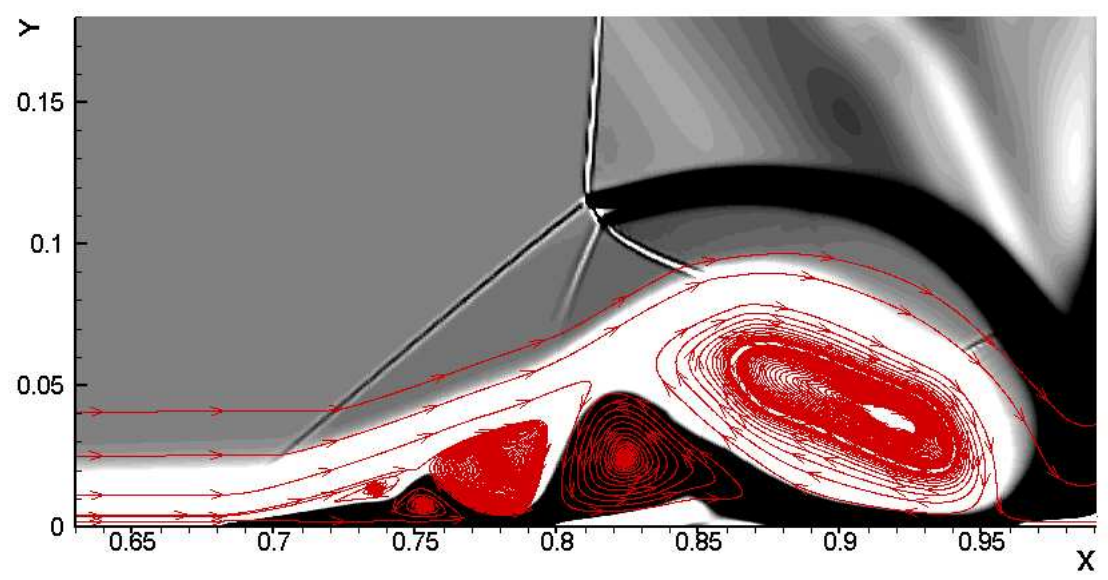

(b)

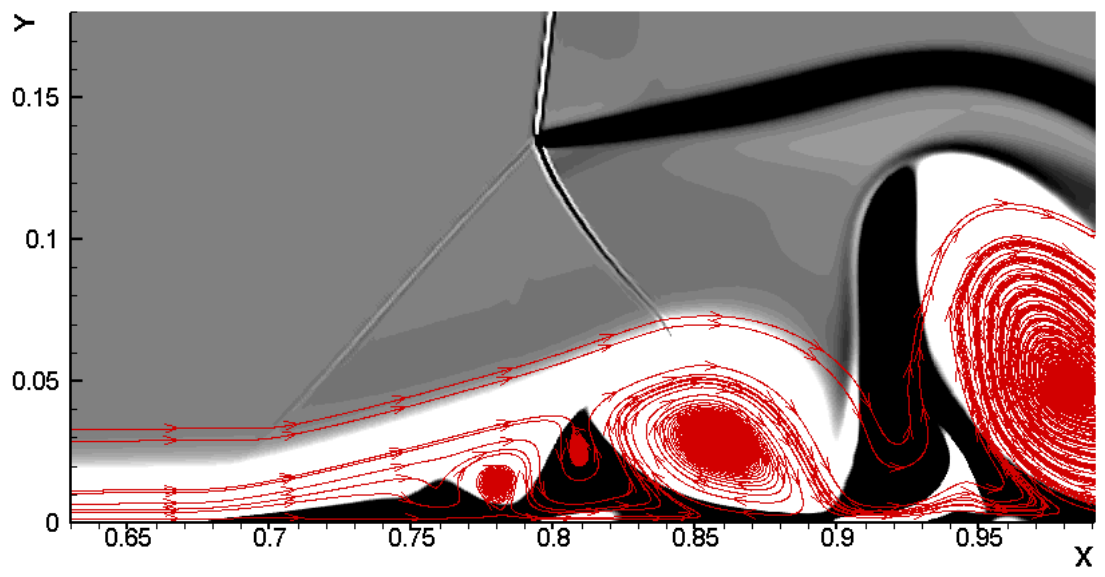

(c)

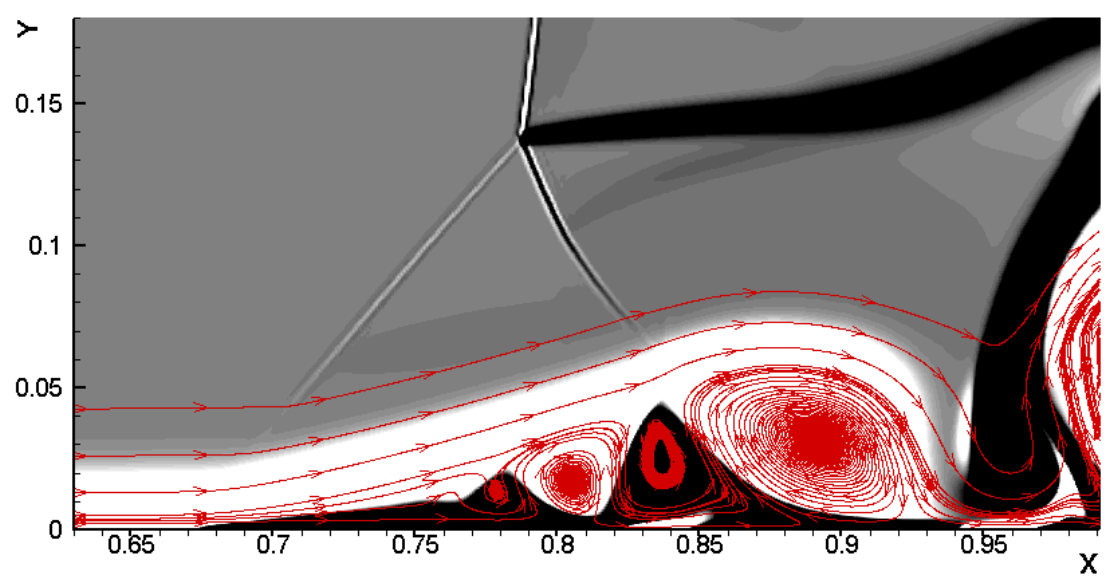

Fig. 10. Contours of the vorticity field with streamlines superimposed obtained, in a coordinate referential attached to the reflected normal shock, for $R e=200$ at time (a) $t=0.6$, (b) $t=0.9$ and (c) $t=1$. 
(a)

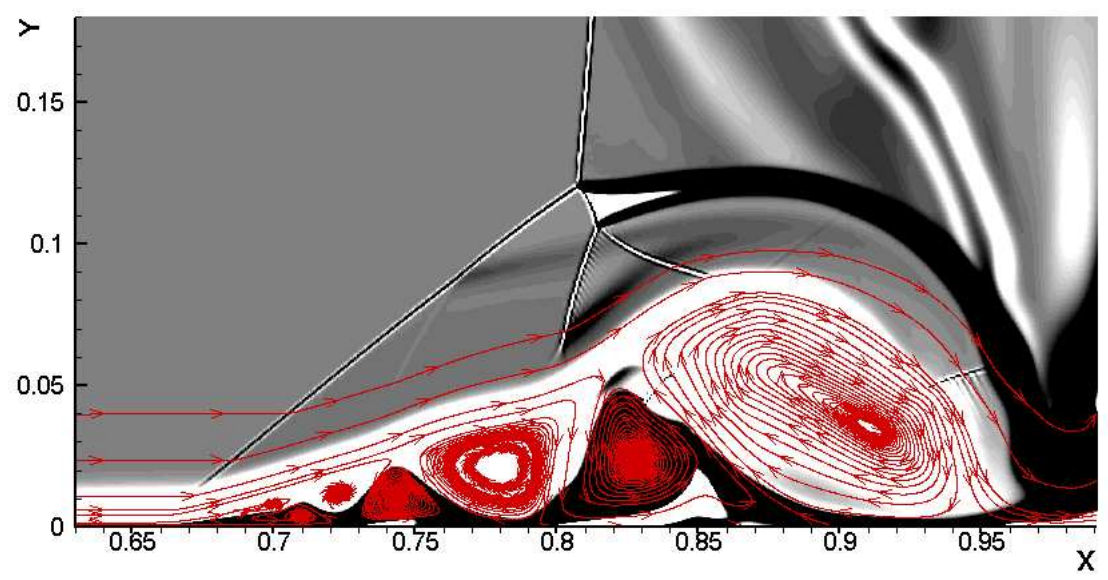

(b)

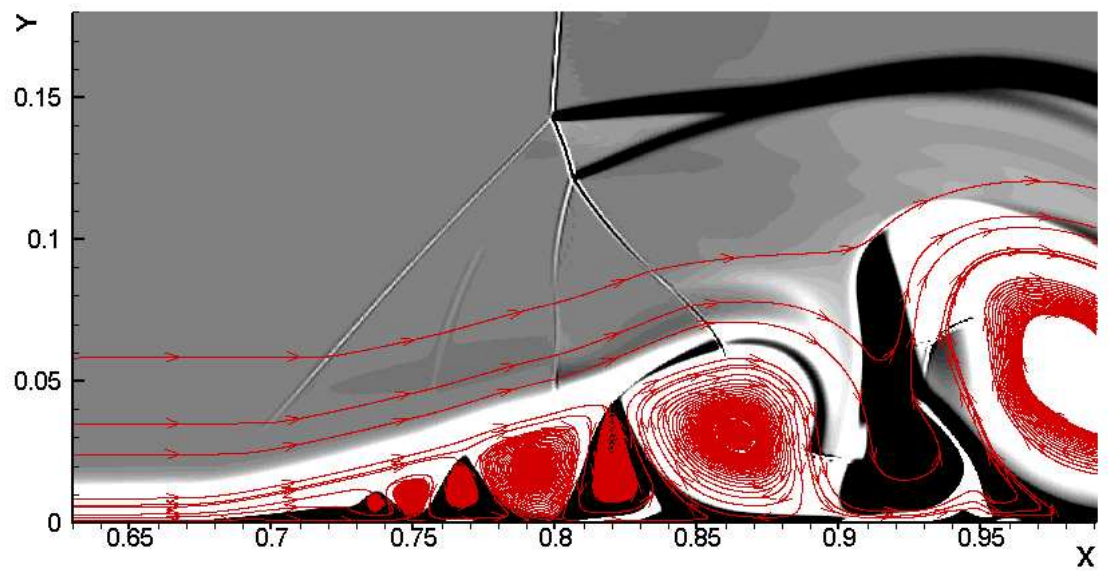

(c)

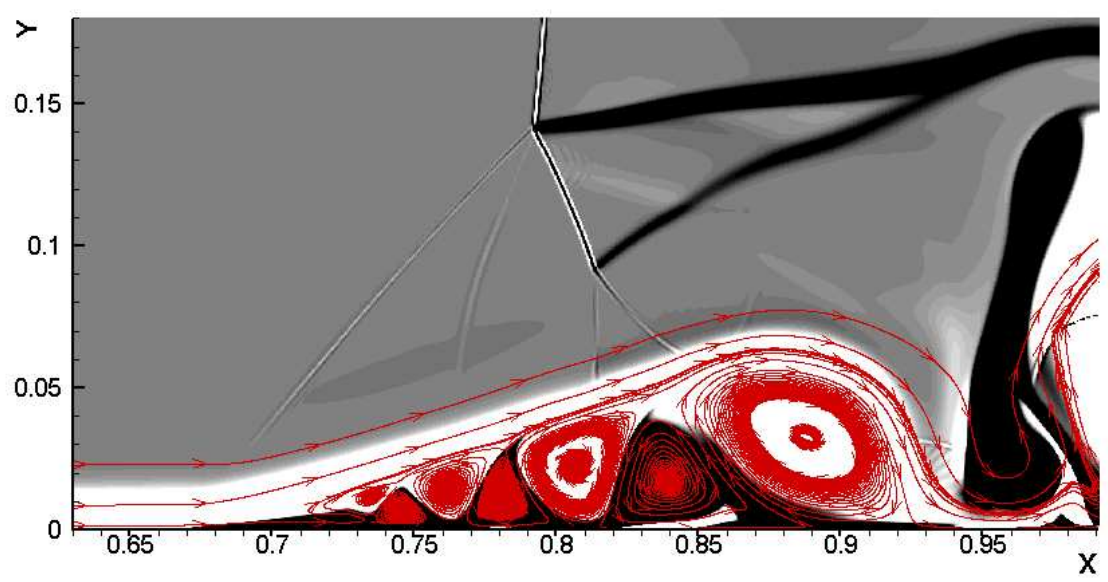

Fig. 11. Contours of the vorticity field with streamlines superimposed obtained, in a coordinate frame attached to the reflected normal shock, for $R e=500$ at time (a) $t=0.6$, (b) $t=0.9$, (c) $t=1$. 
(a)

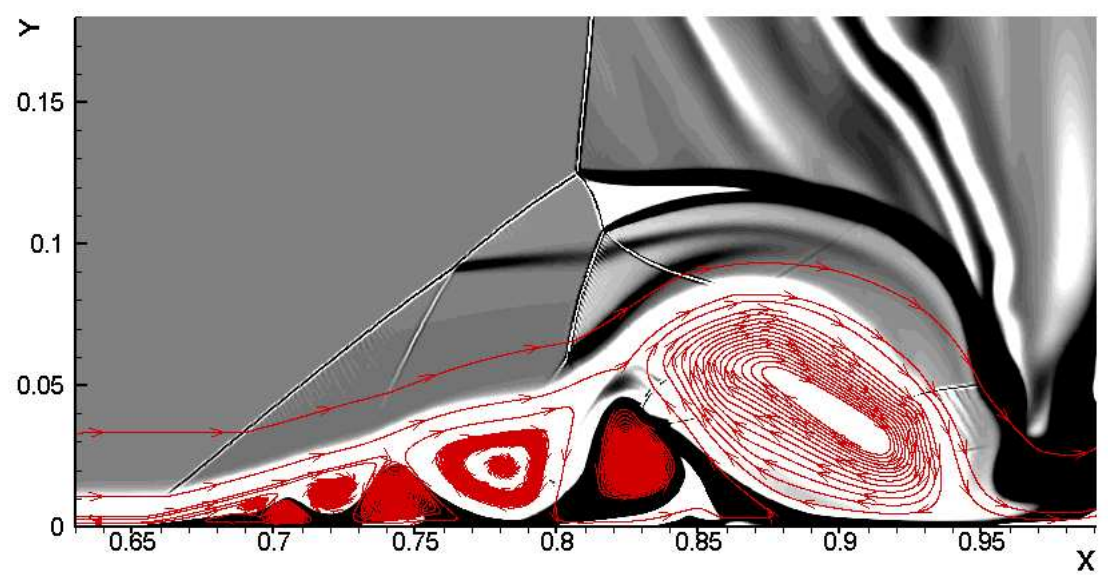

(b)

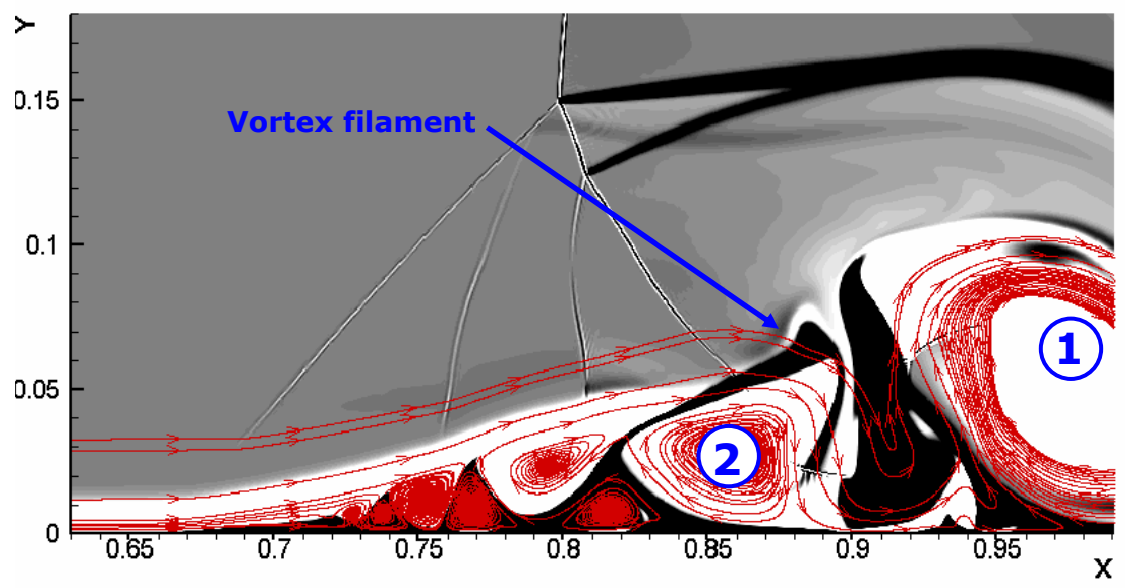

(c)

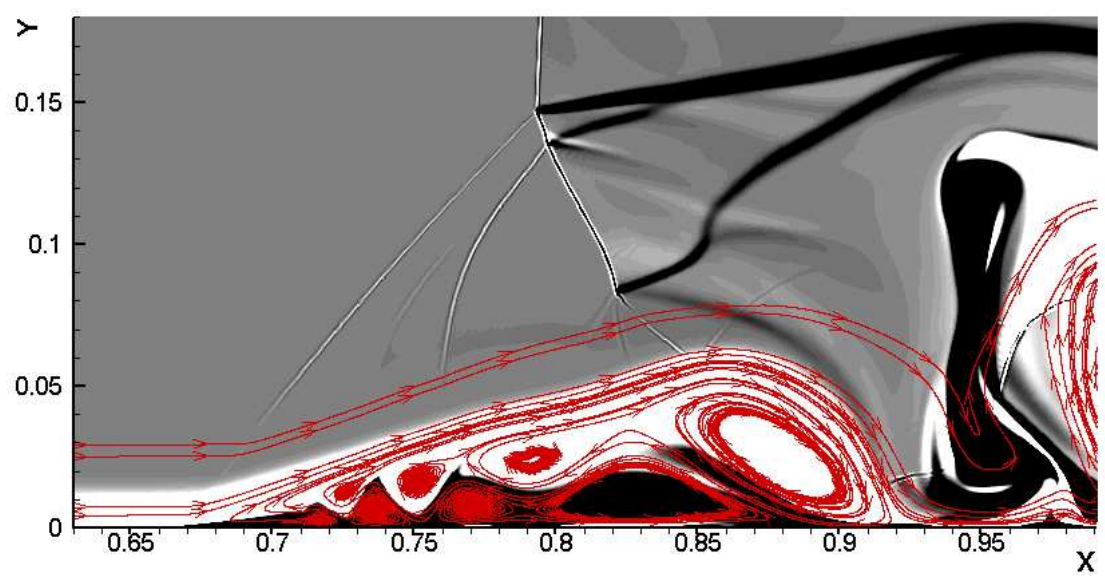

Fig. 12. Contours of the vorticity field with streamlines superimposed obtained, in a coordinate frame attached to the reflected normal shock, for $R e=750$ at time (a) $t=0.6$, (b) $t=0.9$, (c) $t=1$. 
(a)

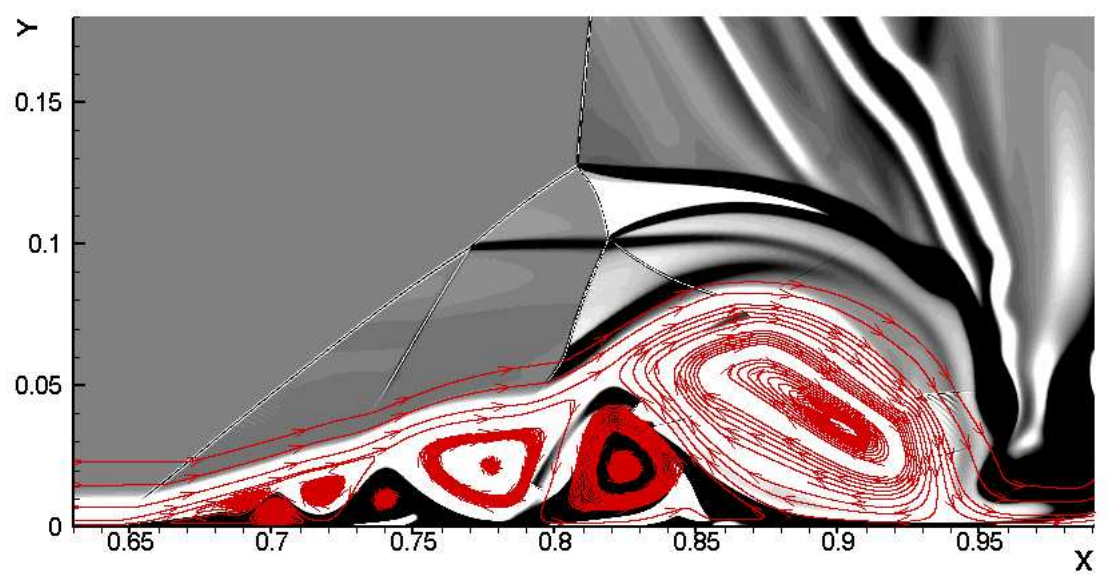

(b)

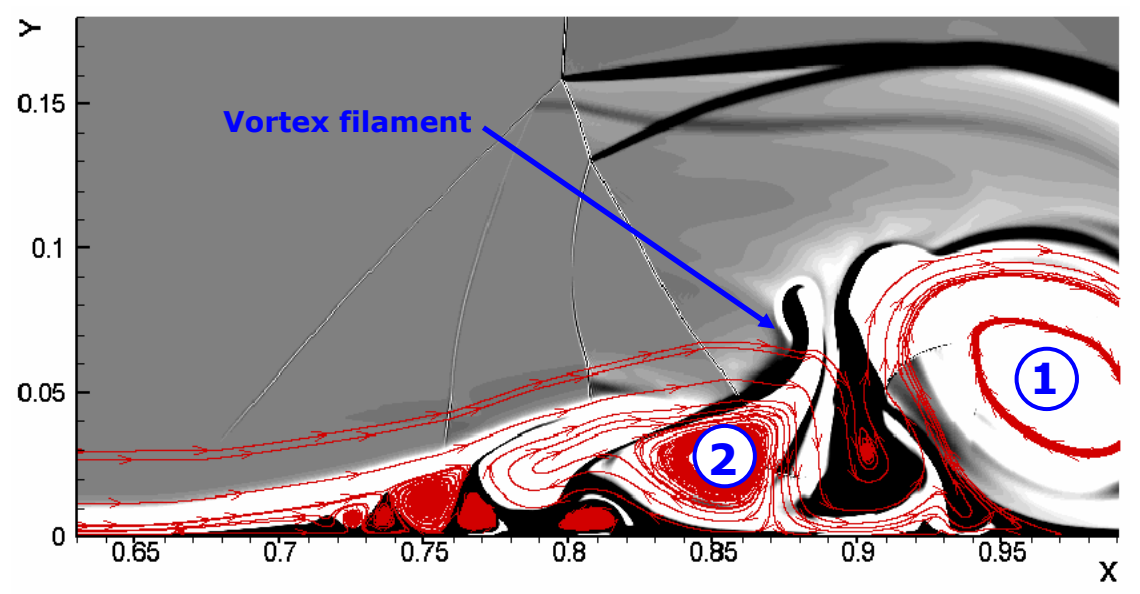

(c)

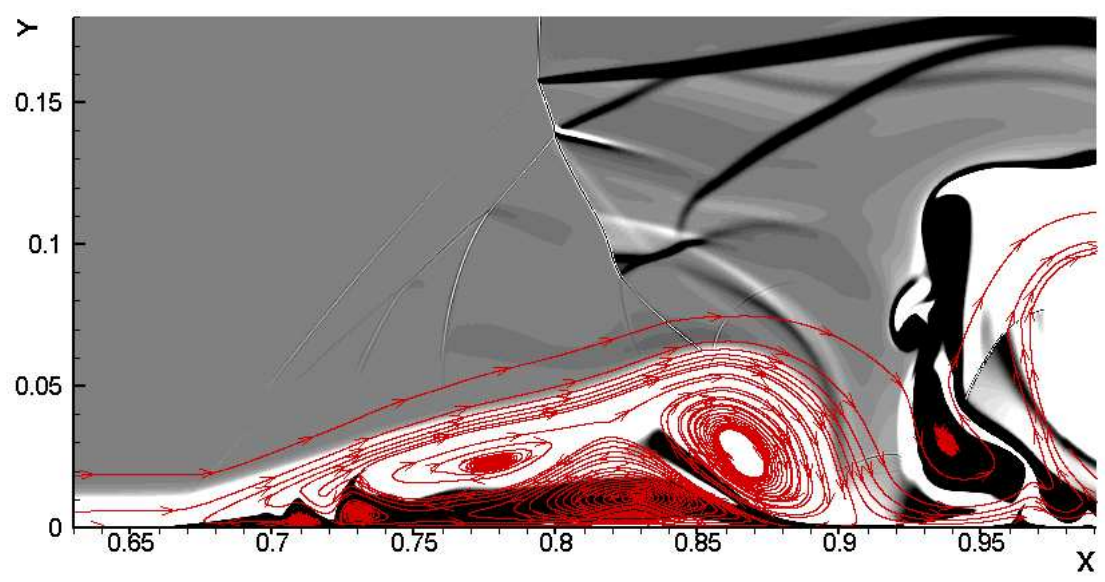

Fig. 13. Contours of the vorticity field with streamlines superimposed obtained, in a coordinate frame attached to the reflected normal shock, for $R e=1000$ at time (a) $t=0.6$, (b) $t=0.9$, (c) $t=1$. 
(a)

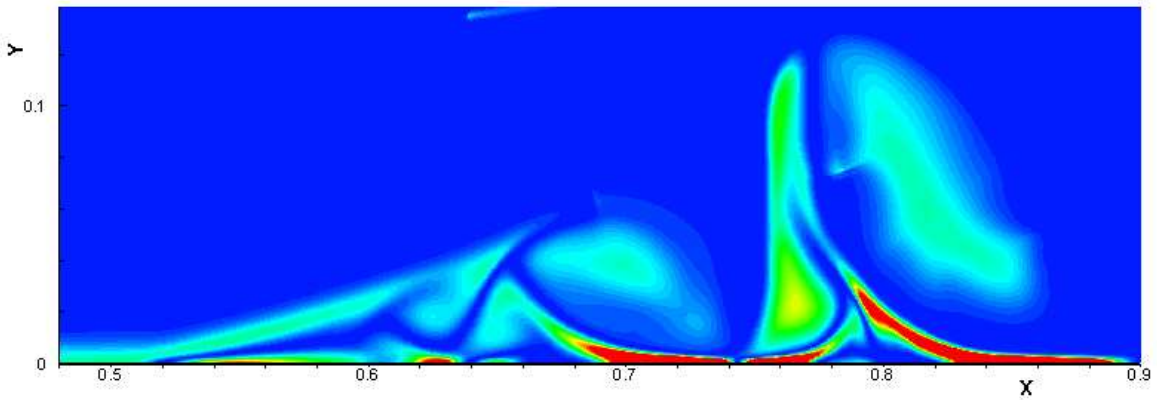

(b)

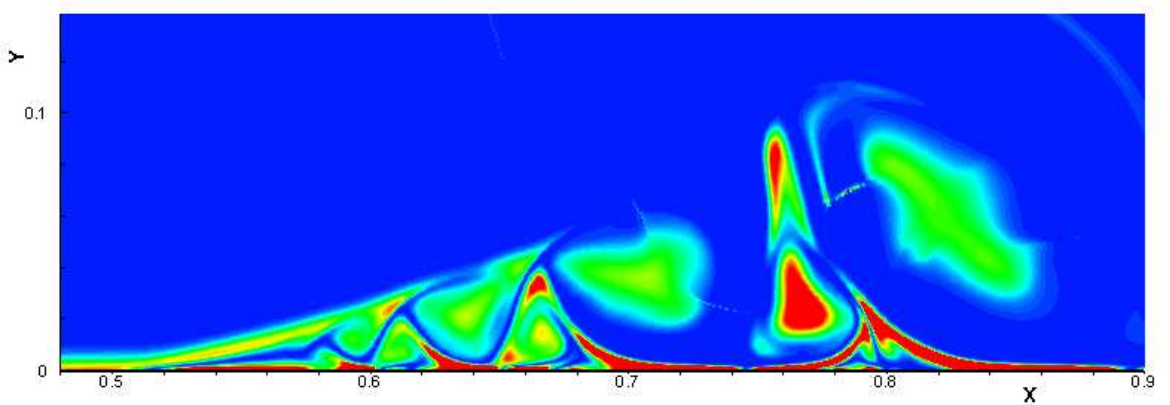

(c)

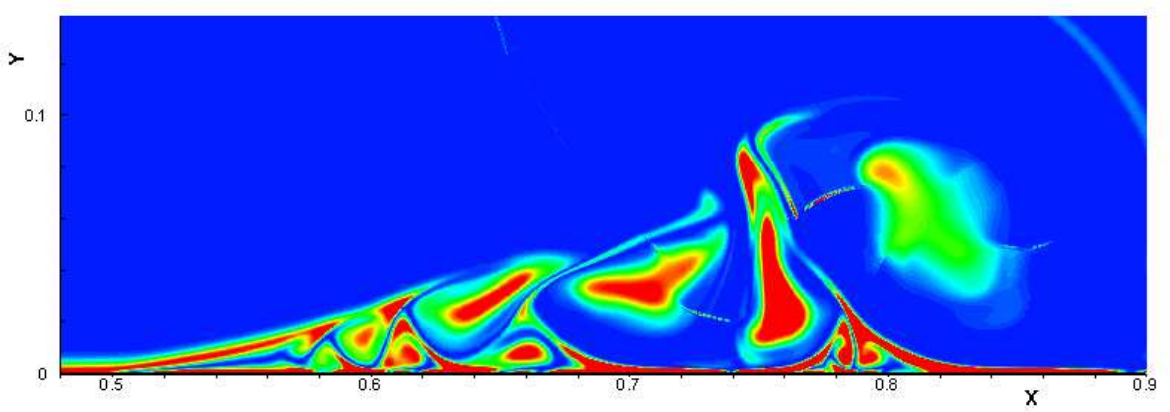

(d)

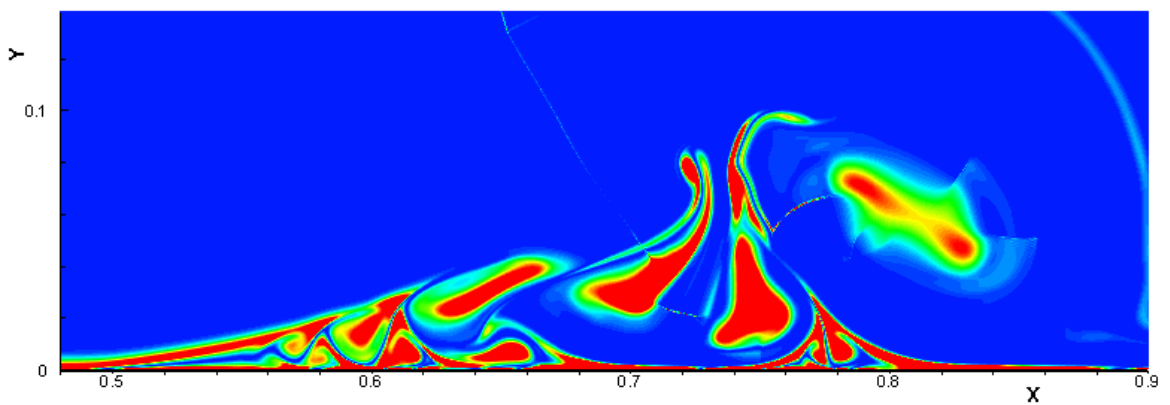

Fig. 14. Contours of enstrophy density $\left(\frac{1}{2}\left|w^{2}\right|\right)$ obtained at time $=0.9$ for (a) $R e=200$, (b) $R e=500$, (c) $R e=750$ and (d) $R e=1000$. 